\title{
Impact of non-linear electronic circuits and switch of chaotic dynamics
}

\author{
M. Lellis Thivagar ${ }^{1}$, Mahmood Ali Ahmed ${ }^{2}$, V. Ramesh ${ }^{3}$, Abdulsattar Abdullah Hamad ${ }^{4}$ \\ ${ }^{1,3,4}$ School of Mathematics Madurai Kamaraj University, Madurai, Tamil Nadu, India \\ ${ }^{2}$ Sharqat College of Engineering Tikrit University
}

\begin{abstract}
Switch-mode power supply is an extremely non-linear system that can inevitably exhibit unpredictable behavior. These control laws may be insufficient for nonlinear systems because they are not robust when the requirements on the dynamic characteristics of the system are strict [10]. Control laws that are insensitive to parameter variations, disturbances, and nonlinearities must be used. In this paper, we have tested the method of the first harmonic, used to analyses servo controls with a nonlinear element, and to predict certain non-linear behaviors. It mainly allows predicting the limit cycles, but also the jump phenomena, the harmonics as well as the responses of non-linear systems to sinusoidal inputs. We apply this method for the prediction of limit cycles and the determination of their amplitude and frequency. We take as an example a Boost converter controlled by current [4]. This system is chaotic when the duty cycle is more significant than 0.5: we then eliminate the chaos by applying the slippery mode command (for the ripple of the output voltage, for the current ripple of the inductance and switching frequency) when the output is periodic (duty cycle less than or equal to 0.5). In this article, we assess that established approach provides the best outcomes: it appears that the preference between the classical mode and the sliding mode depends heavily on the variance domain of the parameters $E, R$, and $I_{r e f}$.
\end{abstract}

\begin{tabular}{l} 
Keywords: Switch, Chaotic dynamic, Non-Liner, current $=I_{\text {ref }}$, Load $R$, Behavior \\
\hline Corresponding Author: \\
Abdulsattar Abdullah Hamad \\
School of Mathematics, \\
Madurai Kamaraj University, Madurai, Tamil Nadu, India \\
E-mail: abdulsatar.a.hamad10519@st.tu.edu.iq; satar198700@gmail.com \\
\hline \hline
\end{tabular}

\section{Introduction}

The discovery of the chaotic dynamics of nonlinear systems dates back to Henri Poincar'e's work on the mechanical mechanics and statistical mechanics, around 1900 [3, 5]. They produced little interest and fell into oblivion. It was not until 1963 that Edward Lorenz, a Massachusetts Institute of Technology meteorologist, brought to light the chaotic nature of the weather conditions and, consequently, the uncontrolled movements of a fluid like the atmosphere. As he sought to determine future weather conditions from initial data on his computer, he found that a small change in the initial data (in the order of one per thousand) resulted in radically different results. After having modeled the movement of air masses through (very simplified) determine-dynamic and fluid mechanics relations, he programmed his computer in a way that to obtain a numerical simulation. At the time, it took a lot of time. One day, in order not to start the calculations again from the beginning, he decided to resume his listing and to enter as initial conditions the values taken during the simulation of the previous day. The computer gave him a five-digit precision, but three significant figures seemed to him more than enough for this kind of physical measurement. He, therefore, truncated these numbers and resumed the calculation. The results that followed were the "decal." First, the simulation seemed to restore the same values. Still, after a while there was nothing to agree, everything happened as if the movement represented by these values changed completely of trajectory and this, because of an approximation of the order of $10^{-4}$. This anecdote [12] is the basis of what is now called chaos: a tiny variation of the initial conditions of a system completely upsets its evolution Lorenz had just highlighted sensitivity to initial 
condition He explained this notion very nicely with the help of the following image the flapping of wings of some butterflies can cause storms to the antipodes Lorenz's discovery intrigued several physicists and mathematicians Poincare's work then came out of the closet and was understood as it should have been for a long time They provided the mathematical backbone that would allow the study of nonlinear phenomena in a new light All of this work laid the new ground for reflex ions about determinism and predictability. In this article, we took the example of a current-controlled Boost Converter whose confusion we removed (now that the service cycle is more than 0.5 ) by applying a sliding mode function. We then developed an iterative application that provides the mathematical expressions of the output voltage $\Delta v, \Delta i$ of the inductance current, and the switching frequency, when the output is periodic (cycle ratio less than or equal to 0.5 ) for both control methods $[1,6]$. Based on previous studies it was explained that the switching power supplies usually emit electromagnetic interference at the switching frequency and its harmonics. The voluntary introduction of chaos into these systems has recently been suggested as a means of reducing these spectral emissions, despite the increase in the ripple of the output voltage [2].

This article mainly explain the control of chaos by the sliding mode command method and performs a comparative study of it with the classical method, even when the system is stable.

\subsection{The study of chaos}

The essence of science is predictability. For example, astronomers may predict the next return of Halley's Comet near the earth, but also the precise moment when the upcoming solar eclipse will occur. Most of the fundamental laws of nature are deterministic; they make it possible to know exactly what will happen, from the knowledge of the current conditions. It is now widely accepted that deterministic and predictable are not synonymous [6]. Since it is impossible to know the initial conditions with perfect precision, long-term forecasting is also true, even when the physical laws are deterministic and exactly known.

A good example is that of meteorology, and deterministic physical laws govern it. Still, their extreme sensitivity to the initial conditions makes long-term predictions difficult. The unpredictable behavior of systems of determinism has been called chaos.

\subsection{Use of chaos}

For many applications, the chaotic response is low or even harmful, hence the need to control chaos:

- In electrical circuits, the chaotic signal can appear in different components

- In the field of physics, the laser sometimes experiences instability, which significantly limits its applicability. Variants of the OGY method then control its chaotic dynamics

- The Belousov-Zhabotinski reaction is the best-known example of a chemical reaction with chaotic behavior. Its control is done by biochemical parameters, by an electric field or by light $[7,8]$.

- In the medical field, the control of certain chaotic cardiac arrhythmias can lead to the design of an intelligent pacemaker. This promising path leads scientists to try a similar strategy to control the chaotic behavior of the human brain in the epilepsy phase.

- It has even been possible to control a sizeable spatial-temporal system model such as the El Nino phenomenon in the Pacific, although the OGY method is usually applied to low order systems [9].

\subsection{The objective of this work}

We have chosen to study the DC-DC converters: these strongly nonlinear circuits can be made chaotic, depending on their parameters. Moreover, the possibility of verifying our theoretical results by the realization of models as well as an economic and industrial realm (cut-off power supplies are omnipresent). In the consumer electronics industry, there have also been essential arguments: in designing reliable power converters, it is vital to be able to assess whether chaotic behavior may occur. It is necessary to be able to study this phenomenon, to avoid it by control of the chaos, even to use it by the generation of the disorder. 


\section{Experimental}

\subsection{Boost converter: control of the current by the classical method}

Figure1 shows the diagram of a currently controlled Boost converter [8]. In this circuit, the current $i$ in the inductance $L$ increases when $S$ is closed (which corresponds to the state of the controlled switch). When the current is reaching the current reference $I_{\text {ref }}$, the switch $S$ opens. The state of the switch $S$ changes according to the clock pulses: if it is open, the first clock pulse changes its state, which becomes closed; if it is closed, then the clock pulses are ignored.
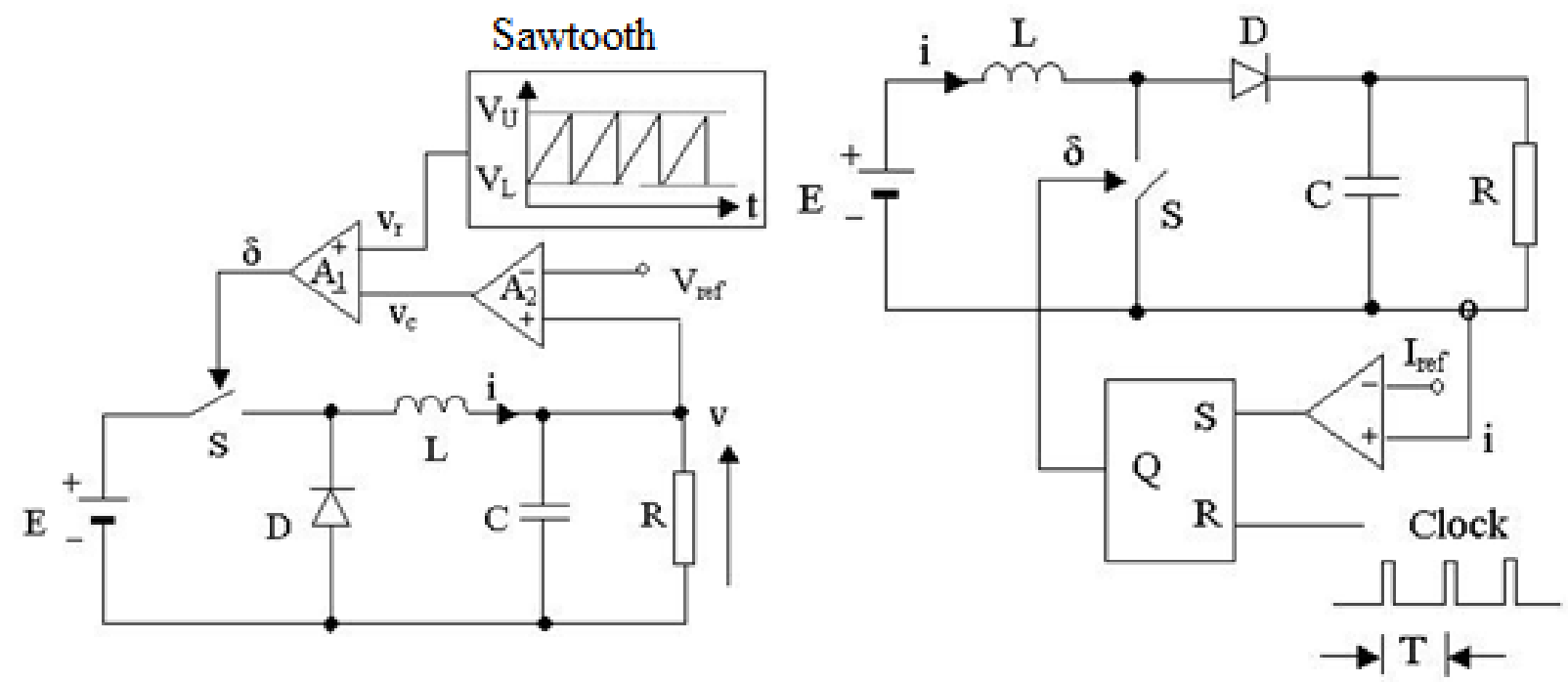

Figure 1. Left Figure: The voltage-controlled Buck converter is a source of nonlinearities. It contains reactive elements (C and L), switching components ( $\mathrm{S}$ and $\mathrm{D})$, and nonlinear control circuits. Figure on the right: The current-driven Boost converter is a source of nonlinearities. It contains reactive elements $(\mathrm{C}$ and $\mathrm{L})$, switching components (S and D), and nonlinear control circuits (the comparator and a digital corrector)

\section{Results}

The differential equations describe the evolution of the variables $i(t)$ and $v(t)$ during the opening of the switch S

$$
\begin{gathered}
L \frac{d i}{d t}+v=E \ldots \ldots \ldots(E q 1) \\
C \frac{d v}{d t}+\frac{v}{R}=i \ldots \ldots \ldots(E q 2)
\end{gathered}
$$

In the other case (during the closing of the switch) the evolution of the variables $i(t)$ and $v(t)$ is described by the differential equations:

$$
\begin{aligned}
L \frac{d i}{d t} & =E \ldots \ldots \ldots(E q 3) \\
C \frac{d v}{d t} & =-\frac{v}{R} \ldots \ldots \ldots(E q 4)
\end{aligned}
$$

To see the behavior of the converter according to these parameters, we adopt modeling of the data in the form of iterative stroboscopic applications: the variables $i(t)$ and $v(t)$ are observing at each clock pulse. The behavior of the Boost converter changes according to the following bifurcation parameters: reference current $I_{\text {ref }}$, input voltage $E$, and load $R$.

Figs. 2 and Figure 3 shows the stroboscopic bifurcation diagrams as a function of $\mathrm{E}$ (the bifurcation parameter) and $I_{r e f}$. The bifurcation diagrams are determined by the superimposition of all the existing in 
points at each clock pulse, eliminating all the values of $i(\mathrm{t})$, which are on the linear parts of this current In all the bifurcation diagrams of the parametric space we delimit zones in which the qualitative behavior of the system is similar for some of the values of the input voltage $\mathrm{E}$ (see Figure. 2, left), the current of the inductor $i$ is a periodic signal of period $1(\mathrm{E}>1.9 \mathrm{~V})$; for other values of $\mathrm{E}$, a zone with a period 2 appears $(1.8 \mathrm{~V}<\mathrm{E}$ $<1.9 \mathrm{~V}$ ) In the same figure, we also see a very rapid sequence of period doublings that leads to chaos, when $\mathrm{E}$ is less than $1.8 \mathrm{~V}$

Decreasing E to $1.9 \mathrm{~V}$ causes the first bifurcation point to appear. For this parameter value, the duty cycle is 0.5 , determining the instability of the converter. The bifurcation diagrams show that an increase in $I_{\text {ref }}$ widens the domain where the periodic signal is of period 1, as well as the zone of instability (which $\mathrm{S}$ includes the chaotic behavior).

The effects of $I_{\text {ref }}$ is synthesizing in the figure. 4, where three areas have highlighted an area where the system does not switch, a zone of stability where $i$ is a periodic signal of period one and, finally, the zone instability for a duty cycle greater than 0.5 . Figs. 5 and 6 show the influence of the parameter $R$ on the bifurcation diagrams: the increase in $I_{r e f}$ decreases the domain where the periodic signal is of period 1. Figure. 7 shows the delimitation of these three zones according to the load.

The purpose of this chapter is to design a command that assures a periodic current of period 1, irrespective of the value of $I_{\text {ref }}$ the zone of instability will thus have been eliminating and the district of stability extended.
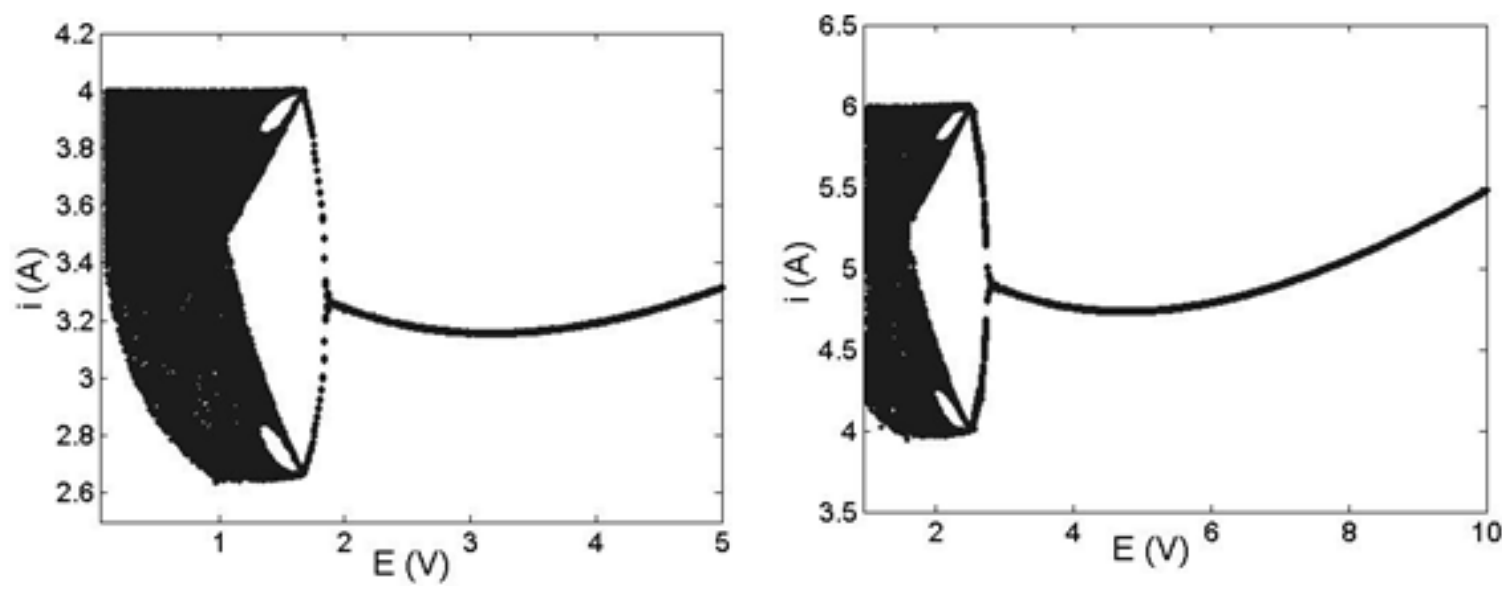

Figure 2. Figure on the left: The bifurcation diagram according to the bifurcation parameter $\mathrm{E}$ for $I_{\text {ref }}=4 \mathrm{~A}$ and $\mathrm{R}=2 \Omega$. Right figure: The bifurcation diagram according to the bifurcation parameter $\mathrm{E}$ for $I_{\text {ref }}=6 \mathrm{~A}$ and $\mathrm{R}=2 \Omega$
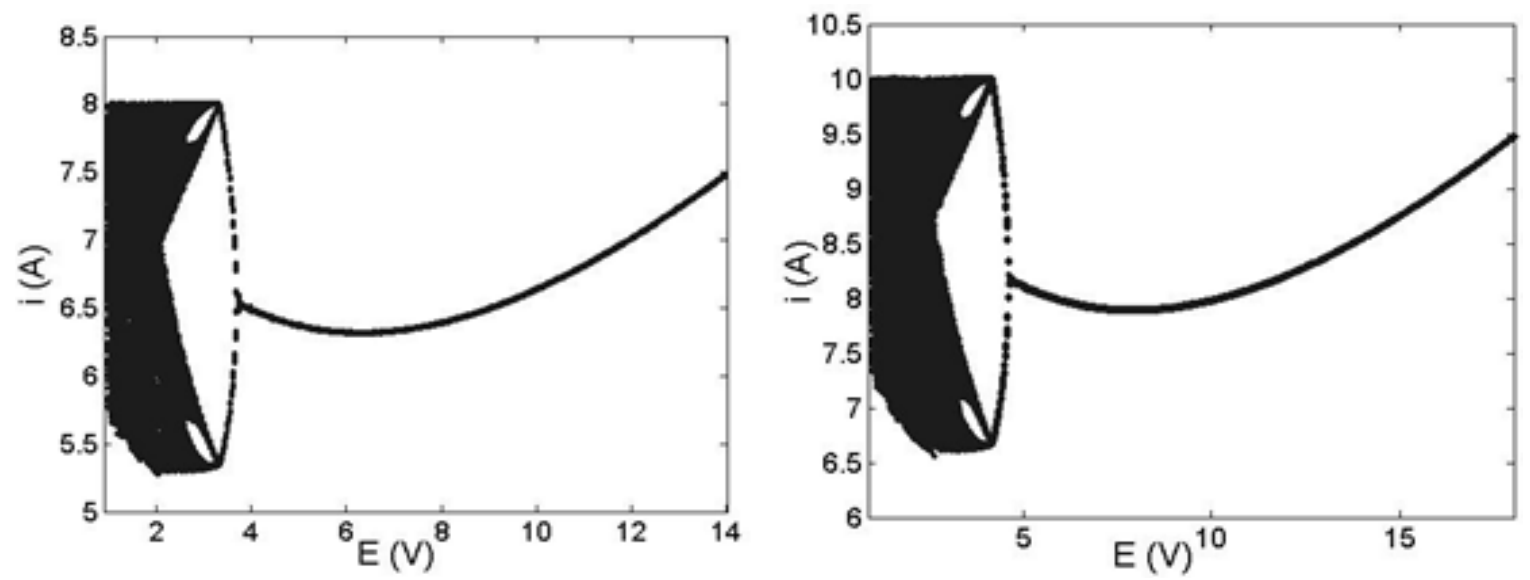

Figure 3. Figure on the left: The bifurcation diagram according to the bifurcation parameter $\mathrm{E}$ for $I_{\text {ref }}=8 \mathrm{~A}$ and $\mathrm{R}=2 \Omega$. Right figure: The bifurcation diagram according to the bifurcation parameter $\mathrm{E}$ for $I_{\text {ref }}=10 \mathrm{~A}$ and $\mathrm{R}=2 \Omega$ 


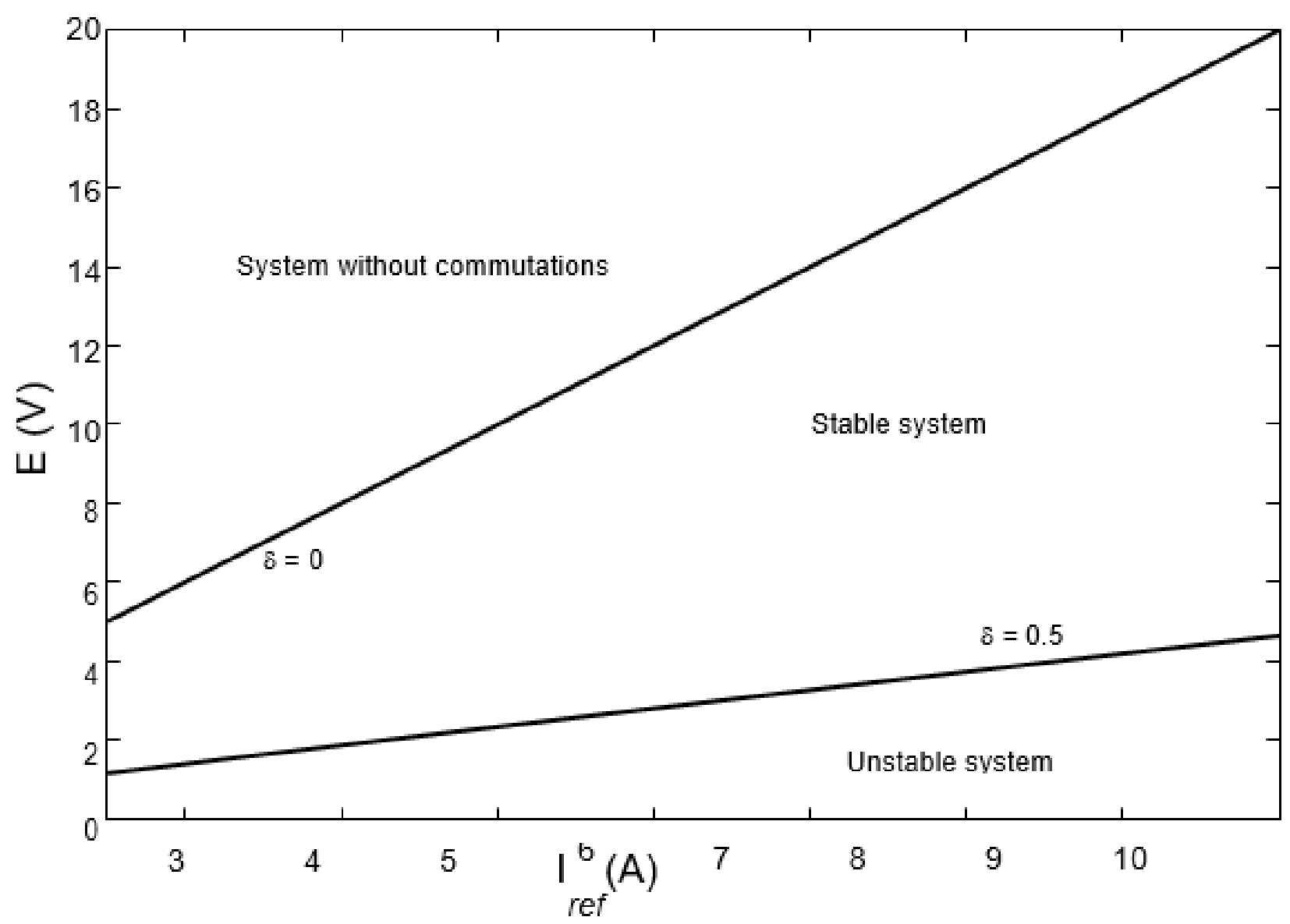

Figure 4. The operating zones of the Boost converter according to $I_{r e f}$ and the bifurcation parameters E, keeping the value of the constant load $\mathrm{R}=2 \Omega$

\subsection{Boost converter: current control by sliding mode control}

The classical control laws of the integral proportional type give good results in the case of linear systems with constant parameters. For nonlinear systems or with non-constant settings, these classical control laws may be insufficient because they are not robust, especially when precision requirements and other characteristics are required. The dynamics of the system are strict. We must then appeal
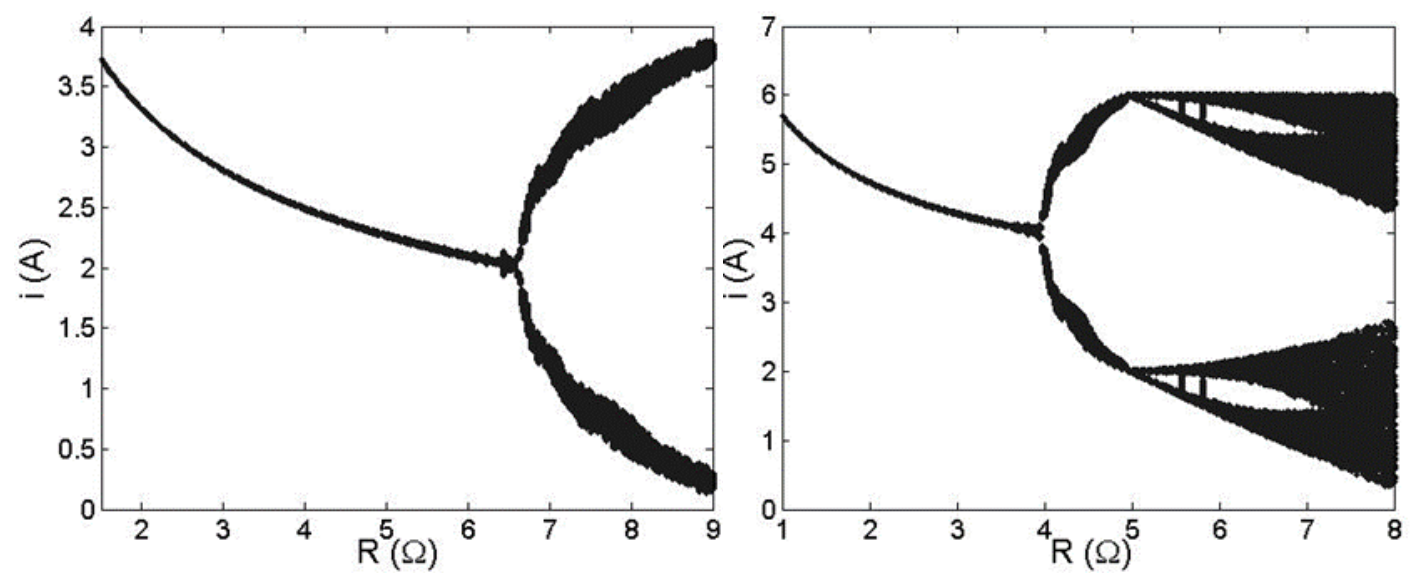

Figure 5. Left Figure: The Bifurcation Diagram Based on the Bifurcation Parameter $R$ for $I_{\text {ref }}=4 \mathrm{~A}$ and $E=$ 5V. Right Figure: The Bifurcation Diagram According to the Bifurcation Parameter $R$ for $I_{\text {ref }}=6 \mathrm{~A}$ and $E=5$ $\mathrm{V}$ 

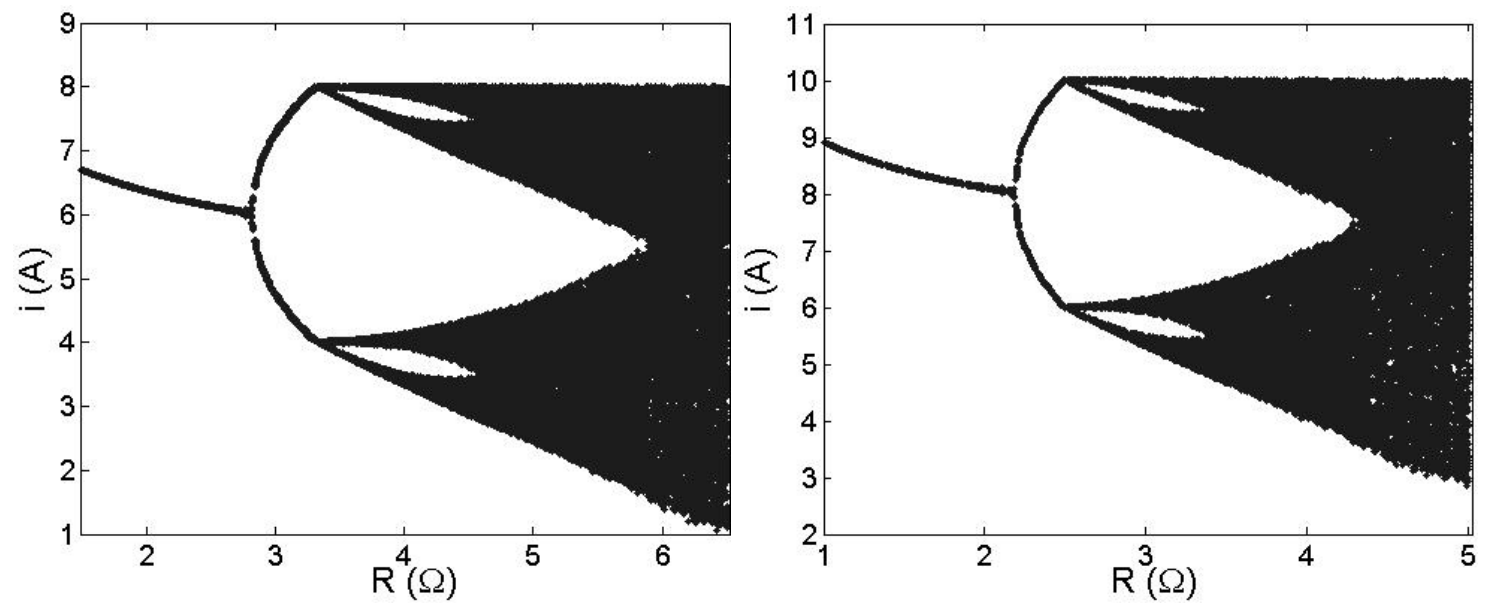

Figure 6. Left Figure: The Bifurcation Diagram Based on the Bifurcation Parameter $R$ for $I_{\text {ref }}=8 \mathrm{~A}$ and $E=$ $5 \mathrm{~V}$. Right Figure: The Bifurcation Diagram According to the Bifurcation Parameter $R$ for $I_{\text {ref }}=10 \mathrm{~A}$ and $E=$ $5 \mathrm{~V}$

Has control laws insensitive to parameter variations disturbances, and nonlinearities. Variable structure control laws are an excellent solution to these problems. The variable structure command is, by nature, a nonlinear command. The main characteristic of systems with variable structure is that their control law changes in a discontinuous manner [13-14]. The switching of the control is performed according to the state variables, used to create a variety of hypersurface called slip whose purpose is to force the dynamics of the system to match with that

\subsection{Boost converter: current control by sliding-mode control}

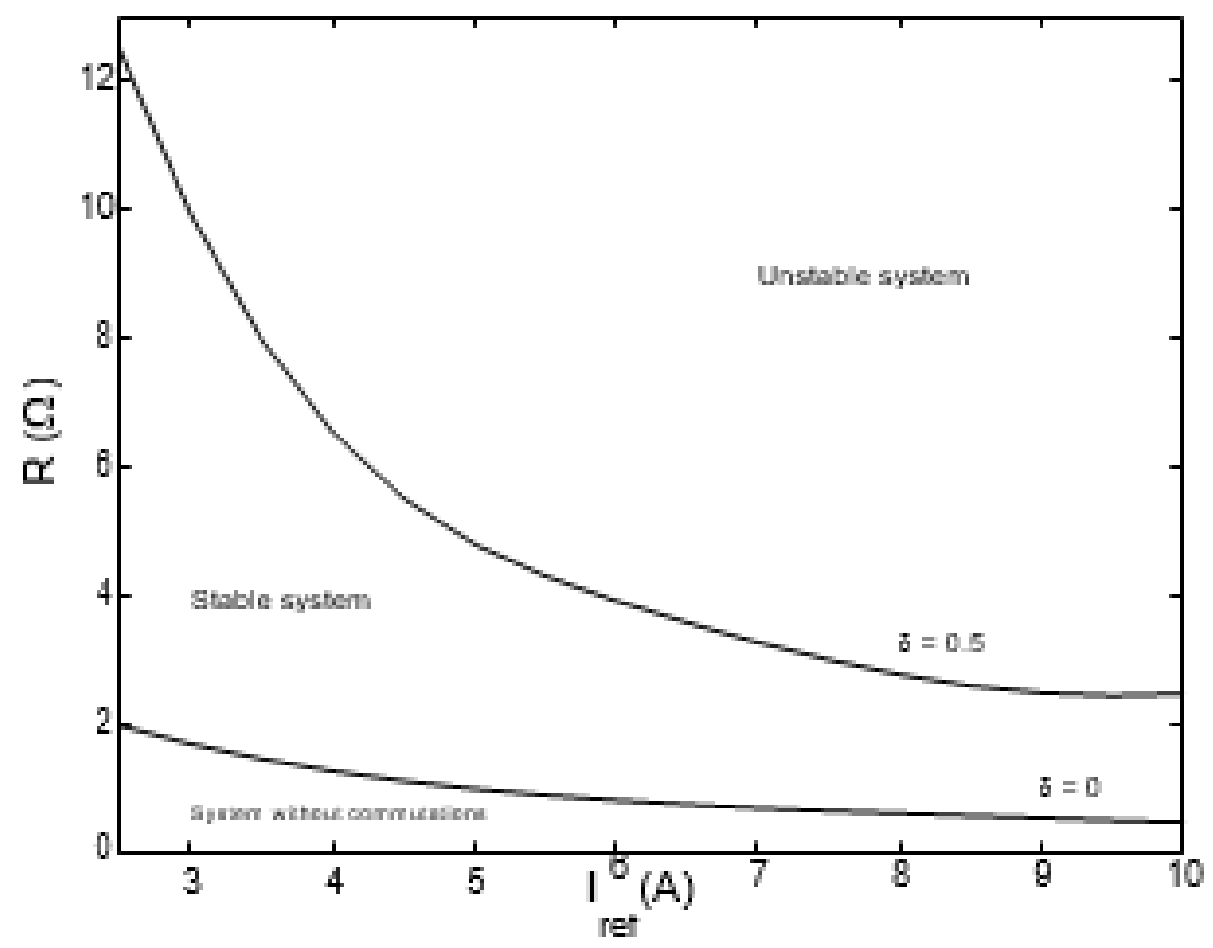

Figure 7. The areas of operation of the Boost converter according to $I_{\text {ref }}$ and the bifurcation parameters $R$, with $E=5 \mathrm{~V}$

The equation of the hypersurface defines it. When the state is maintained this hypersurface, the system is said to be slippery. Thus, as long as slip conditions are ensured the dynamics of the system remain insensitive to 
variations in the process parameters, to modeling errors (in a range which remains broader than that of the approaches conventional automatic) and to certain disturbances This type of control has several advantages such as robustness, high accuracy, stability and simplicity, meager response time This allows it to be particularly adapted to treat systems that have poorly known models, either because of problems of parameter identifications or because of simplification of the model of the system

Differential equations model variable structure control systems with discontinuities (in the second member) due to switching of the control.

The models considered [11] in this account are defined by differential equations of form:

$$
x^{\cdot}(t)=A(x, t)+B(x, t) u(t),(E q . .1 .5)
$$

Where $x \in R^{n}$ is the state vector, $x=\left(x_{1}, x_{2}, \ldots, x_{n}\right)$ and $\mathrm{u} \in R^{m}$ is the control vector of which each component $u_{i}(i=1, \ldots, m)(n>m)$ is a function with discontinuities. Each $u_{i}$ is defined as:

$$
\begin{aligned}
& \left\{\begin{array}{c}
u_{i}(x)=u_{i 0} \text { for } s_{i}(x)>0 \\
u_{i}(x)=-u_{i 0} \text { for } s_{i}(x)<0
\end{array}\right. \\
& i=1,2, \ldots, m . \quad \text { Eq...1.6 }
\end{aligned}
$$

Where $u_{i}(x)=u_{i 0} \operatorname{sign}\left(s_{i}(x)\right.$ for $i=1,2, \ldots, m$.

The control structure is characterized by the sign of a vector function $s(x)$ called a switching function. In the case of linear models, the switching function is chosen as a linear function of the state:

$$
s(x)=[s 1(x) s 2(x) \ldots s m(x)]^{\prime}(E q . .1 .7)
$$

Each scalar switching function $s_{j}(x)=s_{j}\left(x x_{1}, x_{2}, \ldots, x_{n}\right)$ describes a linear $\operatorname{surface}_{j}(x)=0$. The switching surface associated with the system (1.5) defined above:

$$
=\left\{x \in R^{n}: s_{j}(x)=0\right\} \quad j=1,2, \ldots, m .(E q . .1 .8)
$$

Represents a constraint called sliding hypersurface. In the case of linear switching surfaces, it is easy to induce the following conclusions: there are $m$ switching hyper surges $S_{j}$, hence m constraints, and the intersection of all these switching hyper surfaces determines single switching hyper surface. This unique switching hyper surface represents the sum of all $\mathrm{m}$ constraints.

$S=\bigcap_{j=1}^{m} S_{j}=\bigcap_{j=1}^{m}\left\{x \in R^{n}: S j(x)=0\right\}=\sum_{j=1}^{m}\{$ constraints $j\}$. (Eq..1.9)

If, for any initial state vector $\mathrm{x}(\mathrm{t} 0) \in \mathrm{S}$, the state trajectory remains in the hypersurface $S\left(x(t) \in S, \forall t>t_{0}\right)$ then $x(t)$ is a sliding mode for the system.

If every point of $S$ is such that there are state trajectories out of $S$ containing it, then the switching surface $S$ is called the sliding surface.

The purpose of a variable structure control system is to asymptotically bring the state of the system from any initial condition $x(0)=x 0$ to the origin of the space. State when $t \rightarrow \infty$. We consider the sliding mode as defining the motion in the hypersurface $\mathrm{S}$ exclusively, the intersection of all the switching surfaces. This slip mode is often referred to as ideal because it requires an infinitely high switching frequency to exist. Any control system includes imperfections such as delays or hysteresis, which impose a finite commutation frequency. The state trajectory then oscillates in a neighborhood of the sliding surface, and a phenomenon called chattering, as in the figure. 8 .

To illustrate the sliding mode command, let us take as an example the second-order model defined in [4] by the state equations: 

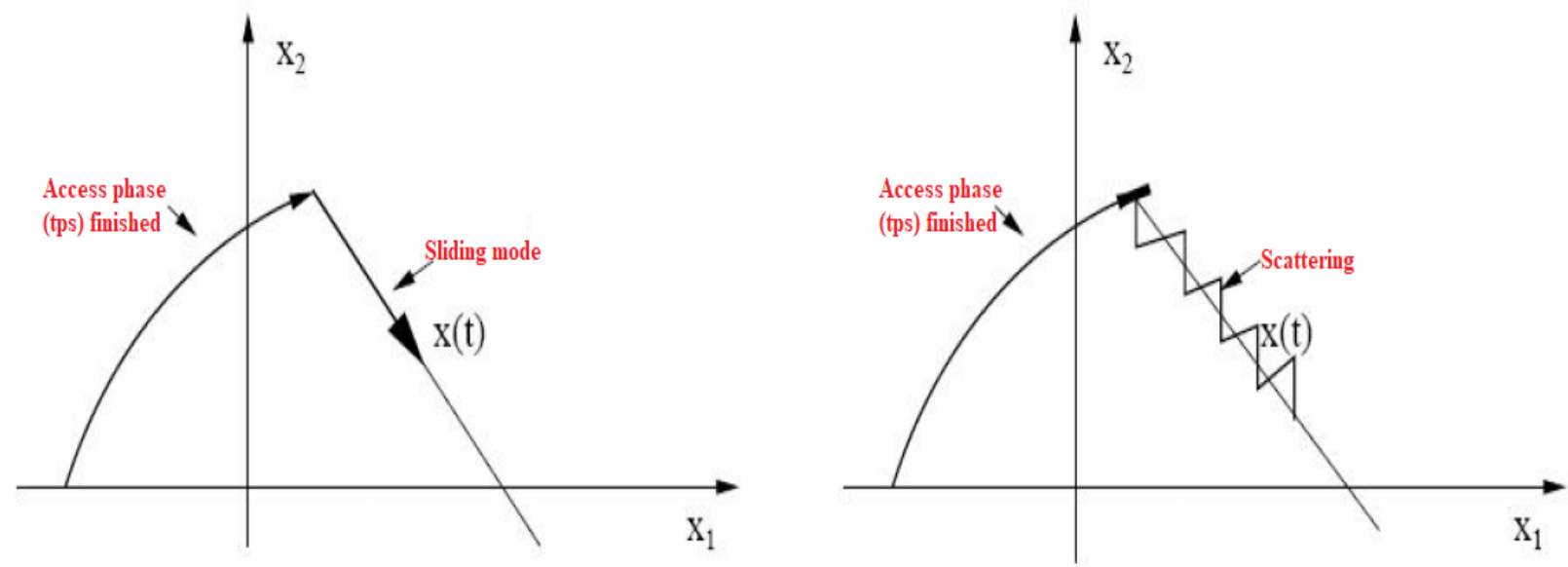

Figure 8. Phenomenon of scattering [13]

$\left\{\begin{array}{l}x_{1}(t)=x_{2}(t) \\ x_{2}(t)=-x_{1}+2 \cdot x_{2}(t)+u(t)\end{array}\right.$

Where $u(t)=-k x 1$ and

$\begin{cases}k=4 & s(x 1, x 2)>0 \\ k=-4 & s(x 1, x 2)<0\end{cases}$

The switching function $\mathrm{s}(x 1, x 2)$ is defined by the quadratic form

$s(x 1, x 2)=x 1(0,5 x 1+x 2) .(1.12)$

In the phase plane, the function $\mathrm{s}\left(\mathrm{x}_{1}, \mathrm{x}_{2}\right)$ corresponds to two lines dividing the plane into regions where the sign of this function changes. The function $\mathrm{s}\left(\mathrm{x}_{1}, \mathrm{x}_{2}\right)$ is called the switching function, while the set of points in the phase plane such that $\mathrm{s}\left(\mathrm{x}_{1}, \mathrm{x}_{2}\right)=0$ is called the switching surface. The control law (here again of state return), therefore, switches for each crossing of the switching surface; the ordered system is thus defined analytically by two different models in two regions of the phase plane. $g$ The first model is given by the following linear state equations

Region I: $\left\{\begin{array}{c}x_{1}(t)=x_{2}(t) \\ x_{2}(t)=-5 x_{1}+2 \cdot x_{2}(t)\end{array}\right.$

The following linear state equations give the second model

Region II: $\left\{\begin{array}{l}x_{1}(t)=x_{2}(t) \\ x_{2}(t)=-3 x_{1}+2 \cdot x_{2}(t)\end{array}\right.$

Region $I$ is defined [Moore. R.1999]s $\left(x_{1}, x_{2}\right)>0$, while region II is defined bys $\left(x_{1}, x_{2}\right)<0$. If we wish to study now the phase trajectories of the global system, it is necessary to add the study of the trajectory of the system on the sliding surface to that in each of regions I and II:

$$
s\left(x_{1}, x_{2}\right)=x_{1}\left(0,5 x_{1}+x_{2}\right)=0 .
$$

The line $\mathrm{x} 1=0$ corresponds to a border where the trajectories of different join, while the line $0,5 x_{1}+x_{2}=0$, $5 x_{1}+x^{*} 1=0$ describes a particular phase trajectory as in figure. 9. When the dynamics of the system is written in this way, it is saying that it is in sliding mode the installation of the limit cycle is governed by four A condition on the state vector defines successive linear differential equations, but who's switching times. 


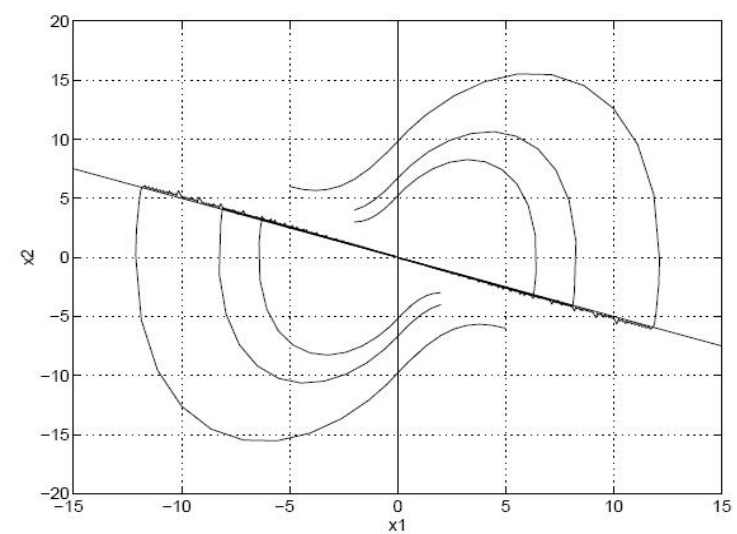

Figure 9. The phase plane with the sliding mode control

When the phase trajectory remains on the surface $S(x)$, the system is said to be in a slippery limit until it reaches a state of equilibrium. This mode of operation corresponds to that of a relay switching at an infinite frequency. An infinite oscillation frequency presupposes elements of good switching (no-threshold relays, hysteresis, or switching delay), which is not the case in practice. In the presence of imperfections (hysteresis, lag), the switching frequency then becomes finite, the oscillation around $\mathrm{S}$ will have an amplitude all the more significant, and a rate all the more low that these imperfections will be necessary.

The theory of the command in slippery mode is a priori well adapted to the control of the converters because it takes into account the discrete nature of these devices. Also, this approach makes it possible to process multivariable systems, while guaranteeing excellent robustness to parametric variations. Finally, it provides excellent dynamic performance. For all these reasons, we chose to apply these techniques to converters.

The sliding mode control is suitable for systems with a variable structure or subsystems with switching logic (this is called discontinuous control) like the power electronics systems. The sliding mode control has been introduced [13] for three-phase converters, motor controls, and other power electronics applications.

Since the power converters are not very sensitive to variations in their parameters, we will study the discontinuous control for a Boost converter (shown in Figure. 10) to eliminate the chaos. For this type of power, the dynamic behavior of the state variables is imposed by the commutations of the system: the trajectories of the system are directed towards a surface in the state space, and there are maintained thanks to commutations the sliding mode command can be represented with a switching surface (a hyper surface of the state space) along which the state action is to be constrained

Figure. 10 shows the diagram of a current-controlled Boost converter whose parameters are: $L=50 \mu \mathrm{H}, C=$ $725 \mu \mathrm{F}, R=2 \Omega, T=400 \mu \mathrm{s}$ and $E=5 \mathrm{~V}$. The model of the converter can be written from equations (1.1) (1.2) (1.3) (1.4) in a restricted form:

$\frac{d i}{d t}=(1-\delta) \frac{1}{L} v+\frac{E}{L} \quad($ Eq...1.16)

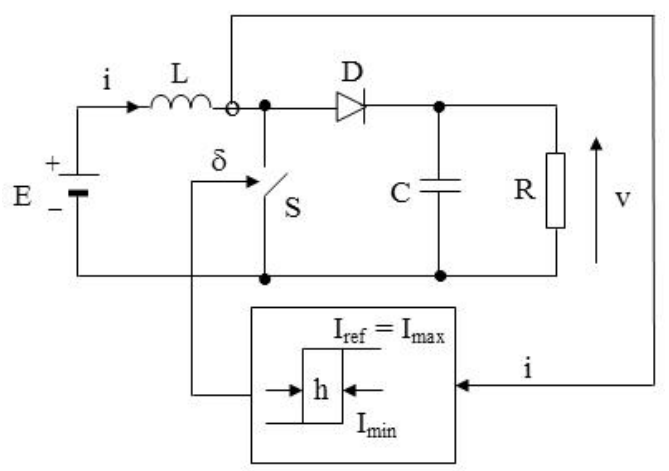

Figure 10. The boost converter controlled by a current with the command a sliding mode (the corrector with hysteresis) 
$\frac{d v}{d t}=(1-\delta) \frac{1}{C} i+\frac{1}{R C} v(\mathrm{Eq} \ldots 1.17)$

The switching right is given by the following relation

$s(i)=i-I * .(\mathrm{Eq} \ldots 1.18)$

Where $I *$ is the desired value of the current $i$. The trajectory in the state space is oriented towards the switching surface

$s(i)=0$ or $i=I * .($ Eq...1.19)

The corrector with the `a slip mode 'command has a discontinuous character and has only two values, 0 and 1 . We can write it as:

$\delta=\left\{\begin{array}{ll}0, & s(i)>0 \\ 1, & s(i) \leq 0\end{array}(\right.$ Eq...1.20)

The condition of existence of the sliding mode is::

$s \cdot s^{\cdot}<0($ Eq...1.21)

From where

$i-I^{*}\left(\frac{E}{L}-(1-u) \frac{v}{L}\right)<0(\mathrm{Eq} \ldots 1.22)$

This relationship can be reduced to a simpler form:

$v>E .(\mathrm{Eq} \ldots 1.23)$

This condition (1.23) means that the sliding mode can be obtained as long as the output voltage $\mathrm{v}$ is greater than $E$ (essential condition for a boost converter). In the regularization procedure, the imperfections of the switching components must be taken into account. This is why we introduced hysteresis $h$ into the implementation of the relay. The limit values of the current $i$ can be calculated:

$\left\{\begin{array}{l}I_{\min }=I^{*}+h \\ I_{\max }=I^{*}-h\end{array}(\mathrm{Eq} \ldots 1.24)\right.$

A simulation of the trajectory in the phase plane leads to the figure. 11 left. After a transient period, the system reaches the limit cycle, so a periodic movement shown in the figure. 11 right.
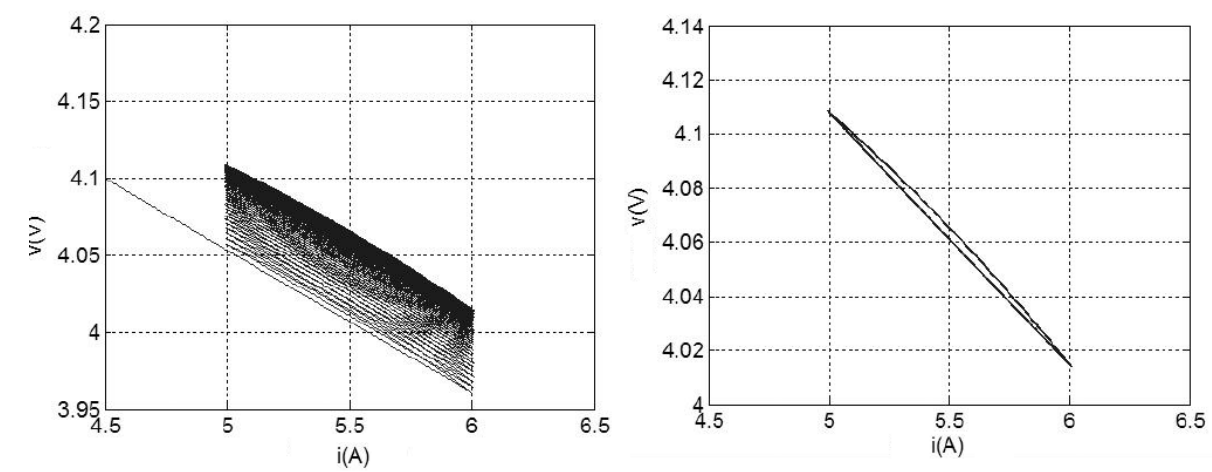

Figure 11. Phase trajectory of the boost converter exhibiting a periodic behavior with the sliding mode control $\left(E=1.5 \mathrm{~V}, R=2 \Omega, I_{\max }=6 \mathrm{~A}, I_{\min }=5 \mathrm{~A}\right)$ 
The difference between the two methods - conventional control and slippery mode control - is the reaction. To show the efficiency of the sliding mode control, let the Boost converter operate in the chaos zone with the conventional power, with $E=1.5 \mathrm{~V}$ and $R=2 \Omega$. In figure. 12, we have represented the response of current $i(t)$ under the conditions stated. For $t \geq 12 \mathrm{~ms}$, the boost converter is forced to operate on a limit cycle using a sliding mode command.

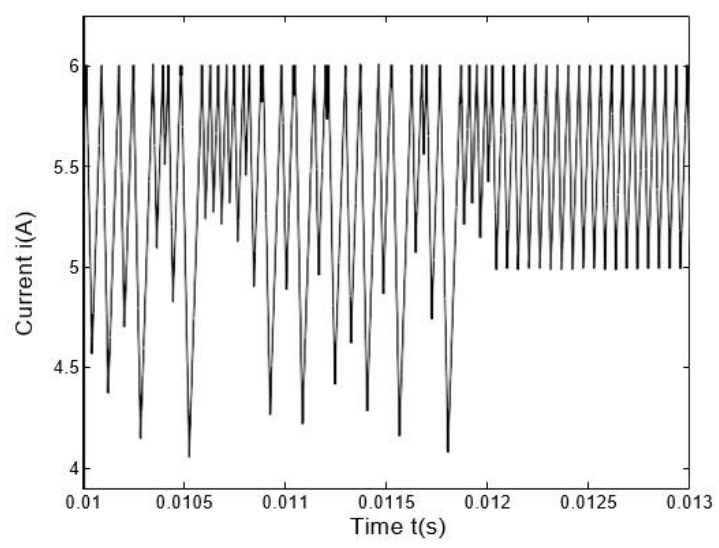

Figure 12. Time trajectory of the current $i(t)$ in the inductance $L$ : chaotic behavior of the Boost converter (t $<12 \mathrm{~ms})$ with the classical method and periodic behavior $(\mathrm{t} \geq 12 \mathrm{~ms})$ with the command a sliding mode $(E=$ $1.5 \mathrm{~V}, R=2 \Omega$ ).

Figure. 13 shows the disappearance of the zone of instability: the area of stability is widened We then eliminate chaos by applying the slippery mode command.
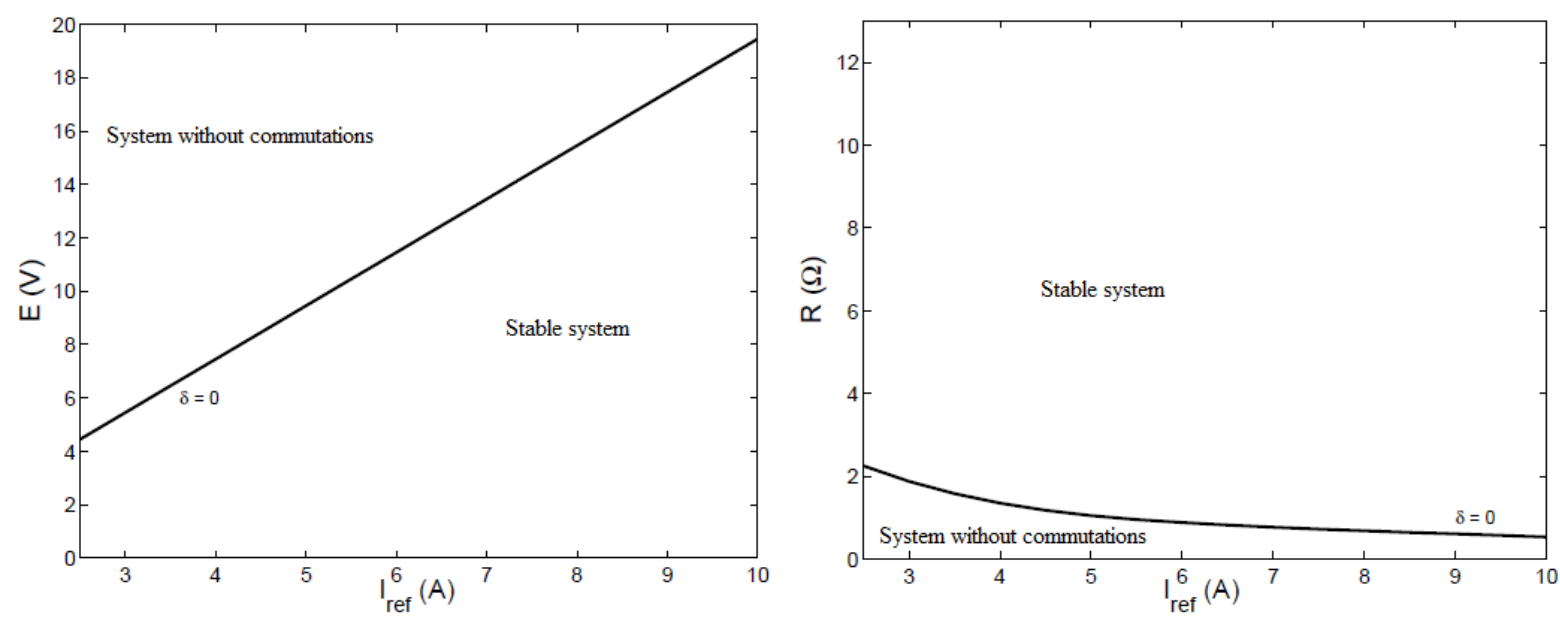

Figure 13. Operating zones of the Boost converter with the 'a sliding mode command as a function of $E$ and $I_{\text {ref }}$, with $R=2 \Omega$ (left figure) then as a function of $R$ and $I_{\text {ref }}$ with $E=5 \mathrm{~V}$ (right picture)

\subsection{Iterative application of the classical method}

We later developed an iterative application to determine which way gives the best results (for output voltage ripple, current ripple, and switching frequency). When the output is periodic (that is, in the stability zone). Figure. 14 represents the time response of the Boost converter operating in the stable area. We have highlighted the two states on and off of the controlled switch $S$, but also the values of the output voltage (in and $\mathrm{v}_{\mathrm{n}+1}$ ) and the current (in and $\mathrm{i}_{\mathrm{n}+1}$ ), necessary to calculate the ripple of the output voltage $\Delta \mathrm{v}$, the current ripple $\Delta \mathrm{i}$, and the switching frequency $f$. The response (Figure. 14) is synchronized to the T period clock, with

$t_{n+1}+t_{n}=T,(\mathrm{Eq} \ldots 1.25)$ 
Which imposes a fixed switching frequency

$f=\frac{1}{T}(\mathrm{Eq} \ldots 1.26)$

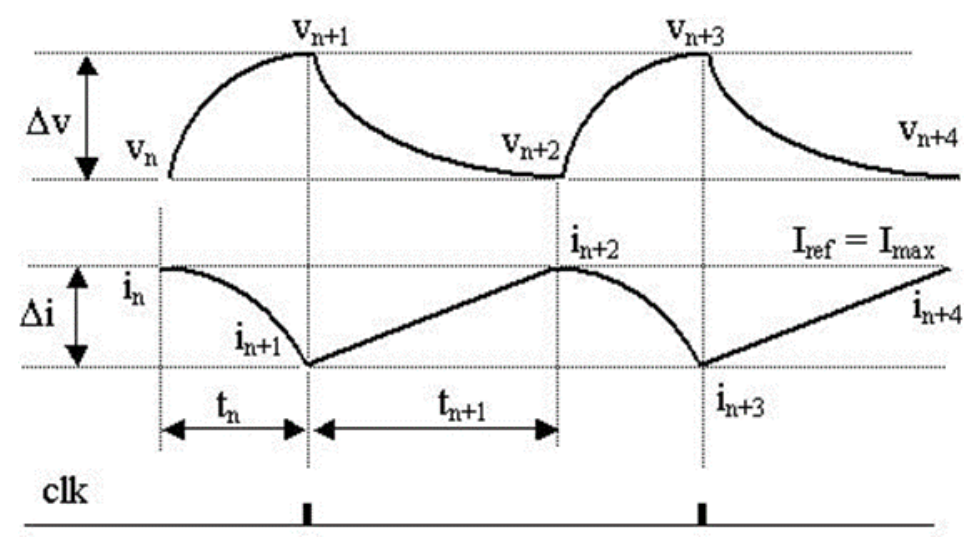

Figure 14. Time trajectories of the current $i(t)$, the output voltage $v(t)$, and the clock pulses of the Boost converter (period $T$ - classical method)

We can then reduce the closing time of the switch $\mathrm{S}: \mathrm{t}_{\mathrm{n}+1}=\mathrm{T}-\mathrm{t}_{\mathrm{n}}$. (Eq..1.27)

The periodic response of the classical method imposes i $(0)=$ in $=I_{\text {ref }}, \mathrm{v}(0)=\mathrm{v}_{\mathrm{n}}$ and $\mathrm{t}(0)=0$ to calculate the iterative application. The solutions of the system (1.1) (1.2) are then:

$$
\begin{aligned}
& v(t)=E+e^{-k t}\left[\left(v_{n}-E\right) \cos (\omega t)+\left(\frac{I_{\text {ref }}-\frac{E}{R}}{\omega C}-\frac{k\left(v_{n}-E\right)}{\omega}\right) \sin (\omega t)\right] \\
& i(t)=\frac{E}{R}+e^{-k t}\left[\left(I_{\text {ref }}-\frac{E}{R}\right) \cos (\omega t)+\left(k \frac{I_{\text {ref }}-\frac{E}{R}}{\omega C}-\frac{v_{n}-E}{\omega L}\right) \sin (\omega t)\right]
\end{aligned}
$$

With $=\frac{1}{2 R C} e t \omega=\sqrt{\frac{1}{L C}-k^{2}}$. Knowing that the final values on this time interval

Are $v\left(t_{n}\right)=v_{n+1}, i\left(t_{n}\right)=i_{n+1}$ and in $=I_{\text {ref }}$, we determine them from eq. (1.28) and (1.29):

$$
\begin{aligned}
& v_{n+1}=E+e^{-k t}\left[\left(v_{n}-E\right) \cos (\omega t)+\left(\frac{I_{r e f}-\frac{E}{R}}{\omega C}-\frac{k\left(v_{n}-E\right)}{\omega}\right) \sin (\omega t)\right] \\
& i_{n+1}=\frac{E}{R}+e^{-k t}\left[\left(I_{\text {ref }}-\frac{E}{R}\right) \cos (\omega t)+\left(k \frac{I_{\text {ref }}-\frac{E}{R}}{\omega C}-\frac{v_{n}-E}{\omega L}\right) \sin (\omega t)\right]
\end{aligned}
$$

Let $v\left(t_{n+1}\right)=v_{n+2}, i\left(t_{n+1}\right)=i_{n+2}$ and $i_{n}+2=I_{\text {ref }}$, as in figure. 14. If one chooses the initial conditions $i(0)$ $=\mathrm{i}_{\mathrm{n}}=I_{\text {ref }}, \mathrm{v}(0)=\mathrm{vn}$, we can express the closing time of the switch $\mathrm{S}$, from the eq. (1.3):

$$
t_{n+1}=\frac{\left(I_{r e f}-i_{n+1}\right) L}{E} E q . .1 .32
$$

Let's explain the current: 


$$
i_{n+1}=I_{r e f}-\frac{E\left(T-t_{n+1}\right)}{L} E q . .1 .33
$$

The eq. (Eq...1.4) gives:

$$
v_{n+2}=v_{n+1} e^{-2 k t_{n+1}}=v_{n+1} e^{-2 k\left(T-t_{n}\right)} \quad \text { Eq..1.34 }
$$

The periodic response of the converter makes that $\mathrm{v}_{\mathrm{n}+2}=\mathrm{v}_{\mathrm{n}}$, then:

$v_{n+1}=v_{n} e^{2 k\left(T-t_{n}\right)} \cdot(E q . .1 .35)$

Replace the expression of the current $\mathrm{i}_{\mathrm{n}+1}$ (Eq..1.33) in the eq. (Eq..1.31) gives:

$I_{\text {ref }}-\frac{E\left(T-t_{n}\right)}{L}=\frac{E}{R}+e^{-k t_{n}}\left[\left(I_{\text {ref }}-\frac{E}{R}\right) \cos \left(\omega t_{n}\right)+\left(k \frac{I_{r e f}-\frac{E}{R}}{\omega}-\frac{\left(v_{n}-E\right)}{\omega L}\right) \sin \left(\omega t_{n}\right)\right](E q . .1 .36)$

Let's explain $\mathrm{v}_{\mathrm{n}}$ based on $\mathrm{t}_{\mathrm{n}}$ alone:

$v_{n}=E+k L\left(I_{\text {ref }}-\frac{E}{R}\right)+\omega L\left(I_{\text {ref }}-\frac{E}{R}\right) \operatorname{coton}\left(\omega t_{n}\right)-\omega L\left(I_{\text {ref }}-\frac{E}{R}\right) \frac{e^{k t_{n}}}{\sin \left(\omega t_{n}\right)}+\frac{\omega\left(T-t_{n}\right) E}{\sin \left(\omega t_{n}\right)} e^{k t_{n}}(E q . .1 .37)$

The ripple of the output voltage $\Delta v$ has the expression:

$$
\Delta v=v_{n+1}-v_{n}=v_{n} \cdot e^{2 k(T-t n)}-1(\text { Eq..1.38) }
$$

Using the notation

$\gamma_{1}=I_{\text {ref }}-\frac{E}{R},($ Eq..1.39)

we determine $\Delta \mathrm{v}$ from eq. (Eq..1.38) and (Eq..1.37)

$\Delta v=\left(e^{2 k(T-t n)}-1\right)\left[E+\gamma_{1}\left(k L+\omega L \operatorname{coton}\left(\omega t_{n}\right)\right)-\frac{\omega e^{k t_{n}}}{\sin \left(\omega t_{n}\right)}\left(\gamma_{1} L-\left(T-t_{n}\right) E\right)(\mathrm{Eq} . .1 .40)\right.$

The current ripple $\Delta i$ has a simpler expression:

$\Delta i=i_{n+2}-i_{n+1}=i_{\text {ref }}-i_{n+i}=\frac{E\left(T-t_{n}\right)}{L}($ Eq..1.41)

The undulations of the output voltage $\Delta v$ and the current $\Delta i$ are expressed as a function of the time interval $\mathrm{t}_{\mathrm{n}}$. This time is identified by replacing the output voltage $\mathrm{v}_{\mathrm{n}+1}$ of the eq. (1.35) in the eq. (1.30)

$v_{n} \cdot e^{2 k(T-t n)}=E+e^{-k t_{n}}\left[\left(v_{n}-E\right) \cos \left(\omega t_{n}\right)+\left(\frac{\gamma_{1}}{\omega C}-\frac{k\left(v_{n}-E\right)}{\omega} \sin \left(\omega t_{n}\right)\right](\right.$ Eq..1.42)

We then obtain the expression:

$\gamma_{1} k L\left(e^{2 k(T-t n)}-1\right)+\gamma_{1} \omega L\left(e^{2 k(T-t n)}+1\right) \operatorname{cotan}\left(\omega t_{n}\right)+E\left(e^{2 k(T-t n)}-1\right)-\frac{\gamma_{1} \omega L}{\sin \left(\omega t_{n}\right)}\left(e^{2 k(T-t n)}+\right.$ $e^{\left.-k t_{n}\right)}+\frac{\omega\left(T-t_{n}\right) E}{\sin \left(\omega t_{n}\right)}\left(e^{k(2 T-t n)}-\cos \left(\omega t_{n}\right)\right)+k E(T-t n)=0(\mathrm{Eq} . .1 .43)$

For a Boost converter controlled by current with the conventional method (which operates for a duty cycle $\delta$ less than 0.5) with the input voltage $E$, the inductance $\mathrm{L}$, the capacitance $C$, a charge $R$, a reference current $I_{r e f}$ and the clock period $T$, we determine a time $\mathrm{t}_{\mathrm{n}}$ with the relation (Eq..1.43). Once the time interval $\mathrm{t}_{\mathrm{n}}$ has been determined, the undulations of the output voltage $\Delta v$ and those of the current $\Delta \mathrm{i}$ are obtained with the eq. (1.40) and the eq. (1.41) respectively. The switching frequency is equal to the clock frequency $T$. 
Another case when the output of the converter is periodic is the particular case, $E=2.5 \mathrm{~V}, I_{\text {ref }}=6 \mathrm{~A}, R=2 \Omega$ shown in Figure. 1.2 where $E=5 \mathrm{~V}, I_{r e f}=6 \mathrm{~A}, \mathrm{R}=5 \Omega$ in Figure. 1.5 right. The response is shown in Figure. 15 is of frequency $2 T$, double that clock frequency. The current i reaches the value $I_{r e f}$ simultaneously with a clock pulse, which determines the interval $t_{n+1}$ (equal to $T$ ). Then, until the appearance of the next clock pulse. $t_{n}$ is also equal to $T$ : $t n=t n+1=T$. (1.44)

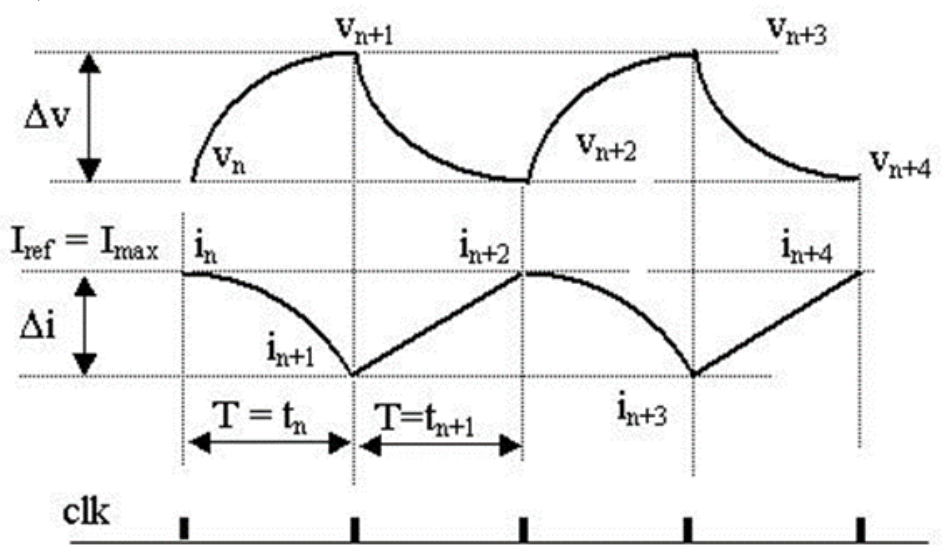

Figure 15. Time trajectories of the current $i(t)$ and the output voltage $v(t)$ and clock pulses of the Boost converter (period $2 T$ - a particular case of the classical method)

The switching frequency is

$f=\frac{1}{2 T}$

For a period of $2 \mathrm{~T}$ (the equation (1.40)) and a fixed time interval $t_{n+1}$ (the equation (1.44)), the current $i_{n+1}$ (the eq. (1.33)).

Therefore:

$i_{n+1}=I_{n+1}-\frac{E T}{L}$

The ripple of the current has the expression:

$\Delta i=I_{\text {ref }}-i_{n+1}=\frac{E T}{L}$

Knowing that $t_{n}$ is given by Eq. (1.44), replacing $i_{n+1}$ of the equation. (1.46) in the eq. (1.31) we obtain:

$I_{\text {ref }}-\frac{E T}{L}=\frac{E}{R}+\mathrm{e}^{-k T}\left[\gamma_{1} \cos (\omega T)+\left(k \frac{\gamma_{1}}{\omega}-\frac{v_{n}-E}{\omega L} \sin (\omega T)\right]\right.$

Identify the output voltage $\mathrm{v}_{\mathrm{n}}$ :

$\left.v_{n}=\frac{\omega L}{\sin (\omega T)}=\gamma_{1}\left(\cos (\omega T)+\left(\frac{k}{\omega} \sin (\omega T)\right)+-\frac{E}{\omega L} \sin (\omega T)-\gamma_{1} \frac{E T}{L}\right) e^{k T}\right]$

Or on the simplified form:

$$
v_{n}=E+\gamma_{1} k L+\gamma_{1} \omega L \operatorname{cotan}(\omega T)-\frac{\omega L e^{k T}}{\sin (\omega T)}\left(\gamma_{1}-\frac{E T}{L}\right)(E q . .1 .50)
$$

For a period of $2 \mathrm{~T}$ (eq (1.40)) and a fixed time interval $\mathrm{t}_{\mathrm{n}}$ (eq (1.44)), the eq. (1.35) becomes:

$$
v_{n+1}=v_{n} e^{k T}(E q . .1 .51)
$$


The ripple of the output voltage $\Delta v$ is expressed as:

$$
\left.\Delta v=e^{2 k T}-1\right)\left[E+\gamma_{1} k L+\gamma_{1} \omega L \operatorname{cotan}(\omega T)-\frac{\omega L e^{k T}}{\sin (\omega T)}\left(\gamma_{1}-\frac{E T}{L}\right)\right](\mathrm{Eq} \ldots 1.52)
$$

\subsection{Iterative Application of Sliding Mode Control}

Figure. 16 represents the transient response of the Boost converter, where we put in the state on (of duration $t_{n}+1$ ) and the off state (of durationt $t_{n}$ ) of the controlled switch. The current control is done with the command a mode sliding, which imposes the constraint (1.19). The current switches between $I_{\max }$ and $I_{\min }$, so this ripple is given by the constant width of hysteresis:

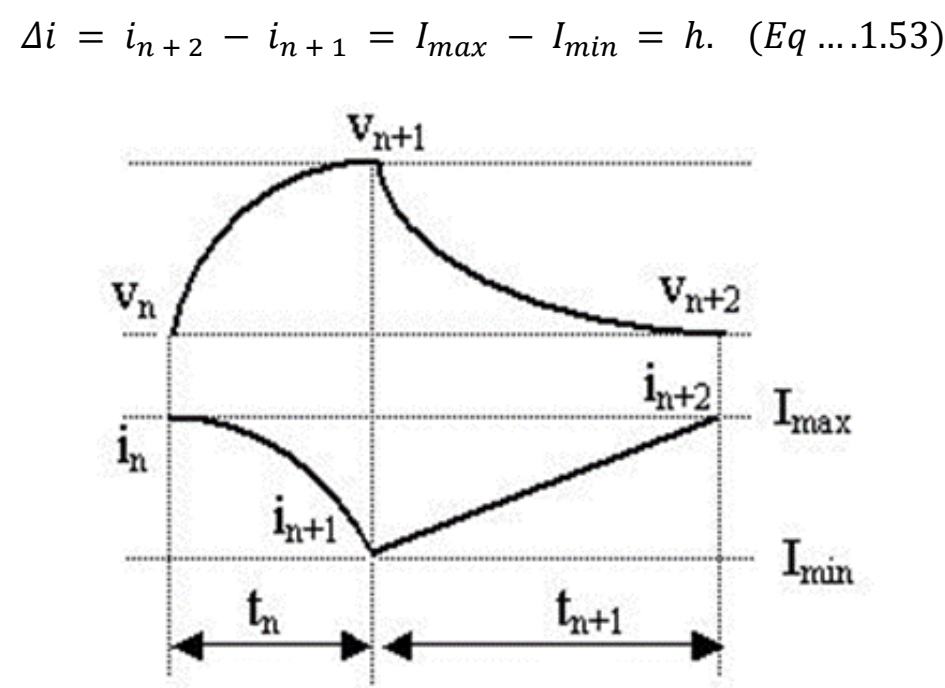

Figure 16. Time trajectories of current $i(t)$ and output voltage $v(t)$ of Boost converter (sliding mode control)

During the off state, the current $i$ is described by the eq. (1.29), where $I_{\text {ref }}$ must replace $I_{\text {min }}$ Taking $i\left(t_{\mathrm{n}+1}\right)=i_{\mathrm{n}+1}=I_{\min }$, we determine the final value of the current $i$ with the relation:

$$
I_{\min }=\frac{E}{R}+e^{-k t_{n}}\left[\left(I_{\max }-\frac{E}{R}\right) \cos \left(\omega t_{n}\right)+\left(k \frac{I_{\max }-\frac{E}{R}}{\omega}-\frac{\left(v_{n}-E\right)}{\omega L}\right) \sin \left(\omega t_{n}\right)\right],
$$

From this relation, we explain the value of the output voltage $v_{n}$ :

$$
v_{n}=E+k L\left(I_{\max }-\frac{E}{R}\right)+\boldsymbol{\omega} \boldsymbol{L}\left(I_{\max }-\frac{E}{R}\right) \operatorname{cost} a n\left(\omega t_{n}\right)-\omega L\left(I_{\min }-\frac{E}{R}\right) \frac{e^{k t_{n}}}{\sin \left(\omega t_{n}\right)} E q \ldots 1.55
$$

The eq. (1.28) with the final value of the output voltage $v\left(t_{n}\right)=v_{n+1}$ and the initial value of the current $i_{n}=$ $I_{\max }$ give the expression:

$$
v_{n+1}=E+e^{-k t_{n}}\left[\left(v_{n}-E\right) \cos \left(\omega t_{n}\right)+\left(\frac{I_{\max }-\frac{E}{R}}{\omega C}-\frac{k\left(v_{n}-E\right)}{\omega}\right) \sin \left(\omega t_{n}\right)\right], \ldots . E q . .1 .56
$$

During the state, the eq. (1.3) and (1.4) are valid. On this interval, $i\left(t_{\mathrm{n}+1}\right)=i_{\mathrm{n}+2}=I_{\min }$ 
The eq. (1.3) makes it possible to determine the duration of this state, knowing that the current $i$ varies between $I_{\max }$ and $I_{\min }$

$$
t_{\mathrm{n}+1}=\frac{\left(I_{\max }-I_{\min }\right) L}{E} \ldots \ldots . E q \cdot .1 .57
$$

Replacing the expression (1.57) in eq. (1.4), we obtain the final value of the output voltage $v_{\mathrm{n}+2}$ :

$$
v_{\mathrm{n}+2}=v_{\mathrm{n}+1} e^{-2 k t_{n+1}}=v_{\mathrm{n}+1} e^{-2 k L^{\frac{\left(I_{\max }-I_{\min }\right)}{E}}} \ldots . E q 1.58
$$

For simplified writing, we use the notation:

$$
\begin{gathered}
a=\frac{2 k L}{E}\left(I_{\max }-I_{\min }\right), E q \ldots 1.59 \\
\gamma 2=I_{\max }-\frac{E}{R}, \ldots . E q 1.60 \\
\gamma 3=I_{\min }-\frac{E}{R}, \ldots . E q 1.61
\end{gathered}
$$

After a state on and a state off, the output voltage $v$ and the current $i$ are periodic, so $v\left(t_{\mathrm{n}+1}\right)=v_{\mathrm{n}+2}=v_{n}$ This allows us to write:

$$
v_{\mathrm{n}+1}=v_{n} e^{a} \ldots E q \cdot .1 .62
$$

The ripple $\Delta v$ has for expression:

$$
\Delta v=v_{n+1}-v_{n}=v_{n}\left(e^{a}-1\right) . . E q . .1 .63
$$

Write this ripple as a function of timet $t_{n}$

$$
\begin{aligned}
\Delta v=\left(e^{a}-1\right) & {\left[E+k L\left(I_{\max }-\frac{E}{R}\right)+\omega L\left(I_{\max }-\frac{E}{R}\right)\right) \operatorname{cotan}\left(\omega t_{n}\right) } \\
& -\omega L\left(I_{\max }-\frac{E}{R}\right) \frac{e^{k t_{n}}}{\sin \left(\omega t_{n}\right)} E q . .1 .64
\end{aligned}
$$

The simplified form is:

$$
\Delta v=\left(e^{a}-1\right)\left[E+k L \cdot \gamma 2+\omega L R I E_{\max } \cot \left(\omega t_{n}\right)-\omega L \cdot \gamma 2 \frac{e^{k t_{n}}}{\sin \left(\omega t_{n}\right)} E q . .1 .65\right.
$$

The switching frequency $f$ is:

$$
f=\frac{1}{T}=\frac{1}{t_{n}+t_{n+1}} E q \cdot .6 .66
$$

or on the explicit form:

$$
f=\frac{1}{t_{n}+\frac{L\left(I_{\max }-I_{\min }\right)}{E}} E q . .1 .67
$$

The ripples of the output voltage $\Delta v$ and the switching frequency $f$ are calculated as a function of the time interval $t_{n}$. This time remains to be identified by replacing the output voltage $v_{n+1}$ of the eq. (1.62) in the eq. (1.56), we get a relation between $v_{n}$ and $t$ : 
$v_{n} e^{a}=E+e^{-k t_{n}}\left[\left(v_{n}-E\right) \cos \left(\omega t_{n}\right)+\left(\frac{{ }^{I} \max -\frac{E}{R}}{\omega C}-\frac{k\left(v_{n}-E\right)}{\omega}\right) \sin \left(\omega t_{n}\right)\right] \ldots . .(E q 1.68)$

From the expression (1.55), it is determined

$$
\begin{aligned}
& k L\left(\gamma_{2} e^{a}-\gamma_{3}\right)+\omega L\left(\gamma_{2} e^{a}-\gamma_{3}\right) \operatorname{cotan}\left(\omega t_{n}\right)+ \\
& +E\left(e^{a}-1\right)-\left(\gamma_{3} e^{k t_{n}} e^{a}+\gamma_{2} e^{-k t_{n}}\right) \frac{\omega L}{\sin \left(\omega t_{n}\right)}=0 .
\end{aligned}
$$

The relation (1.69) allows determining the duration th of the off-state for a boost converter with a sliding mode control, if we know the input voltage $\mathrm{E}$, the inductance $\mathrm{L}$, the capacitance $\mathrm{C}$, the load $\mathrm{R}$ and the variation limits of the current Imax and Imin. Once the time interval tn has been determined the ripples of the output voltage $\Delta v$ and the switching frequency f are obtained with the eq. (1.65) and eq. (1.67) Respectively.

\subsection{Comparison between the two methods}

To find out which method gives the best results (the lowest ripple of the output voltage $\Delta v$ and the current $\Delta i$ and, if possible, the highest frequency $f$ ), we Simulations will be performed simultaneously with the equations (1.40) (1.41) (1.47) (1.52) (classical method) and (1.65) (1.67) (command with sliding mode).

In Figures 17 and 18 are the $\Delta v$ grooves according to the parameter $E$, keeping the charge $\mathrm{R}$ constant and for different values of the current Reference current $=I_{\text {ref }}$.

$\Delta v$ is represented in unbroken lines for the standard order and by circles for individual cases. The undulations of the output voltage $\Delta v$ are shown in thin lines for the sliding mode control for three hysteresis values $h=$ $1 \mathrm{~A}, 1.5 \mathrm{~A}$, and $2 \mathrm{~A}$.

If the hysteresis fails, the ripples of the output voltage $\mathrm{v}$ decrease (for the slippery control mode).

An increasing value of the current reference $I_{\text {ref }}$ determines an increase of $\Delta v$ with the conventional method. This conclusion is also valid for individual cases.

In general, the corrugations $\Delta v$ of the output voltage $v$ of the Boost converter with the conventional method are smaller for low values of the current Reference current $=I_{\text {ref }}$

If this current is large, the command 'a sliding mode gives little ripples $\Delta v$ if the amount of hysteresis is low. In figure. 19 are the dashed $\Delta i$ grooves for the sliding mode control and with an active line for the conventional method. $\Delta i$ decreases if the hysteresis is small and for low values of the reference current $I_{\text {ref }}$. if the benefits of hysteresis
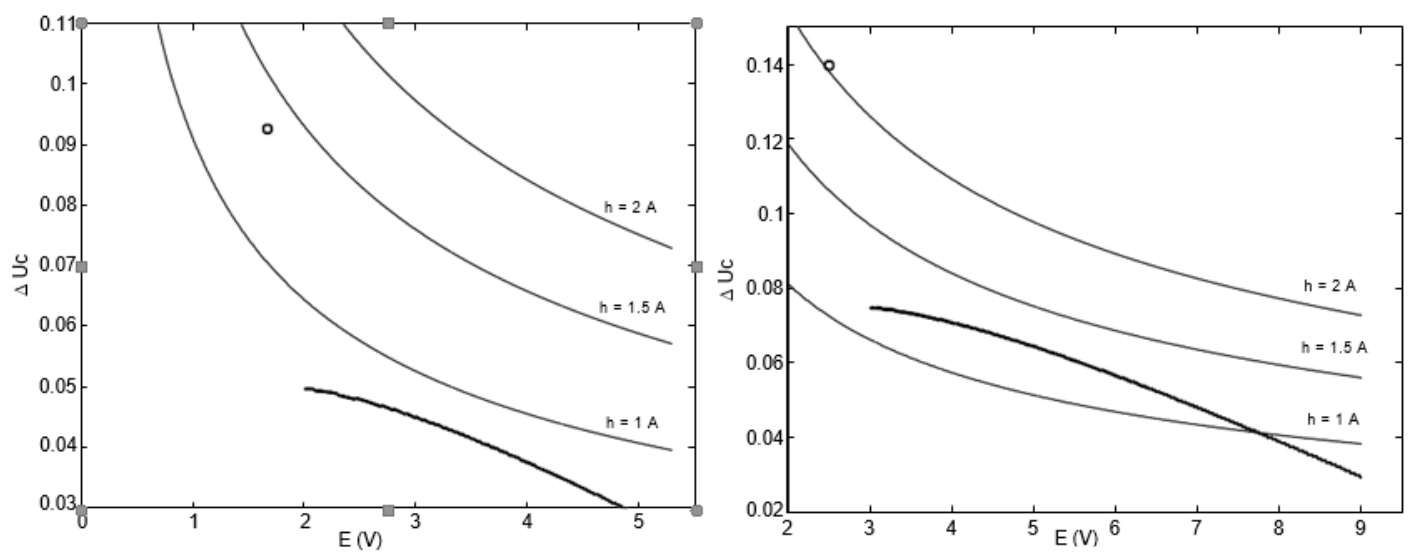

Figure 17. The corrugations $\Delta v$ of the output voltage $v$ of the Boost converter with the classical method (strong strokes - particular case circled) and the control with sliding mode (fine lines) for $R=2 \Omega$ ( $I_{\text {ref }}=4 \mathrm{~A}$ for the figure on the left and $I_{\text {ref }}=6$ A for the number on the right) 

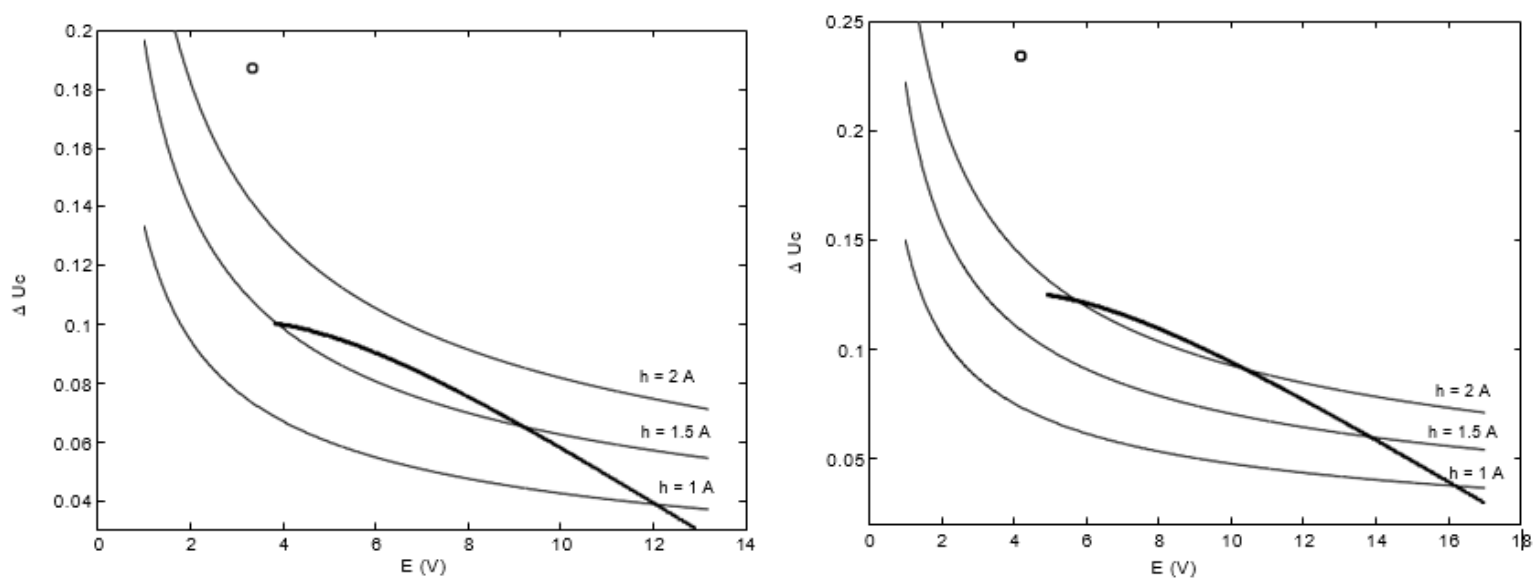

Figure 18. The corrugations $\Delta v$ of the output voltage $v$ of the Boost converter with the classical method (strong strokes - particular case circled) and the control with sliding mode (fine lines) for $R=2 \Omega$ ( $I_{\text {ref }}=8 \mathrm{~A}$ for the figure on the left and $I_{\mathrm{ref}}=10 \mathrm{~A}$ for the picture on the right)

$\mathrm{H}$ And the current reference $I_{\text {ref }}$ is high. The weakest corrugations $\Delta i$ are obtained with one method or the other, depending on the variation domain of the parameters E. Figs. 20 and 21 are represented by the ripple $\Delta v$ as a function of the load $R$, keeping the input voltage $E$ constant and for different values of the current Reference current $=I_{\text {ref }}$

We arrive at the same conclusion as before: if the hysteresis is small, the ripples of the output voltage $v$ are small; use of the sliding mode command is desired For large values of $h$, it is possible to observe the superiority of the classical method, irrespective of the reference current $=I_{\text {ref }}$

Between these two cases, the smallest $\Delta i$ undulations are obtained with one way or the other, according to the range of variation of the load $\mathrm{R}$ the same results are obtained for the undulations

$\Delta i$ represented in Figure. 22

As can be seen in Figs. 20, 21 and 22, the particular case of the classical method does not give good results from the corrugation $\Delta v$, nor even from the creases $\Delta i$

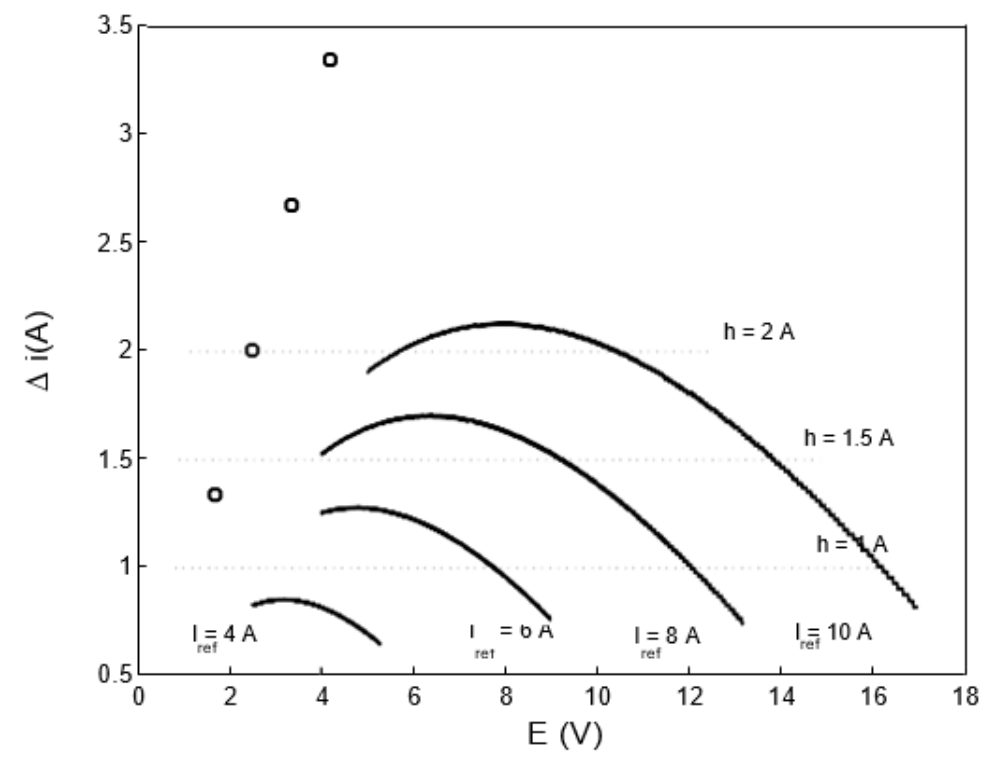

Figure 19. The $\Delta i$ corrugations of the Boost converter with the classical method (strong strokes - particular case circled) and the control with sliding mode (dotted) for $R=2 \Omega$ 

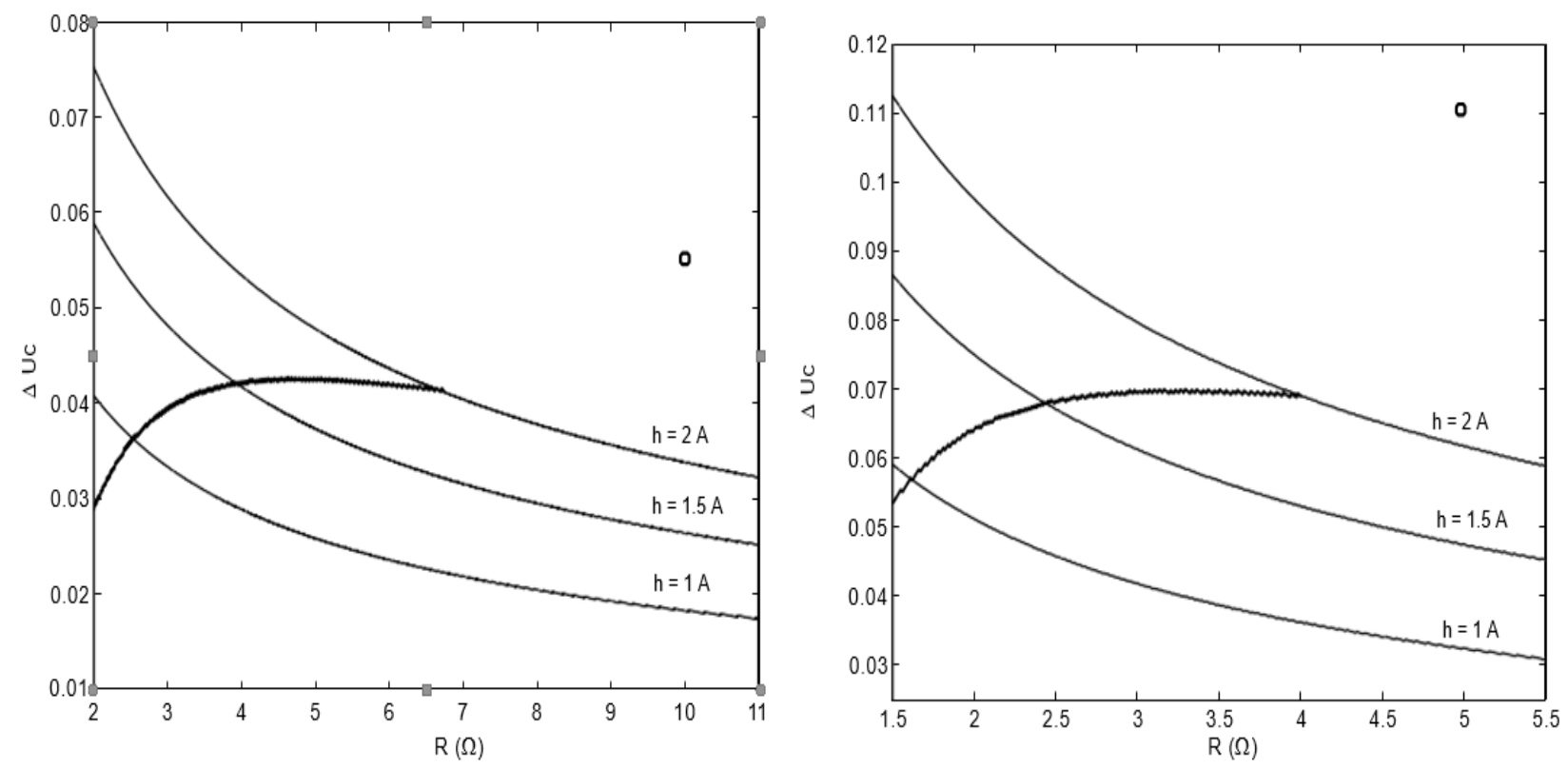

Figure 20. The corrugations $\Delta \mathrm{v}$ of the output voltage $\mathrm{v}$ of the Boost converter with the classical method (strong strokes - particular case circled) and the sliding mode control (fine lines) for $E=5 \mathrm{~V}$ ( $I_{\text {ref }}=4$ A for the figure on the left and $I_{\mathrm{ref}}=6 \mathrm{~A}$ for the figure on the right).

The switching frequency for the classical method has a constant value of $25 \mathrm{kHz}$, and for the particular case of this method, the frequency is reduced by half. These frequencies are shown in Figures-23, 24 inactive lines. For the sliding mode command, the rate is given by the formula (1.67) (in thin lines).

The switching frequency increases for small values of the hysteresis and increases the current reference $I_{\text {ref. In }}$ Figure. 23 left, we see the superiority of the classical method compared to the sliding mode control: higher frequencies and smaller ripples. Increasing the value of the reference current leads to much higher switching frequencies with the sliding mode control, only for low costs of the hysteresis.
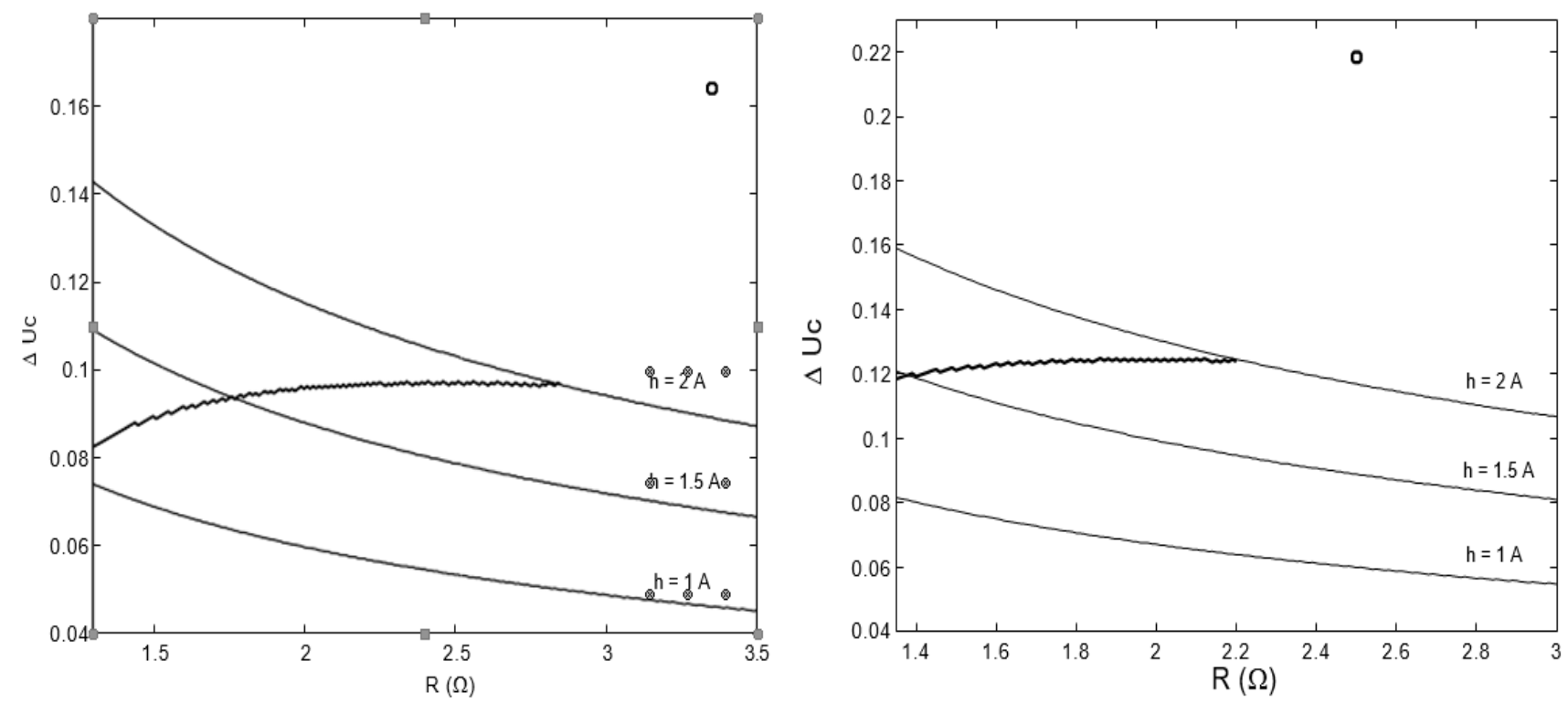

Figure 21. The $\Delta v$ corrugations of the output voltage $\mathrm{v}$ of the Boost converter with the classical method (strong strokes - particular case circled) and the sliding mode control (fine lines) for $E=5 \mathrm{~V}$ ( $I_{\text {ref }}=8 \mathrm{~A}$ for the figure on the left and $I_{\text {ref }}=10$ A for the figure on the right) 


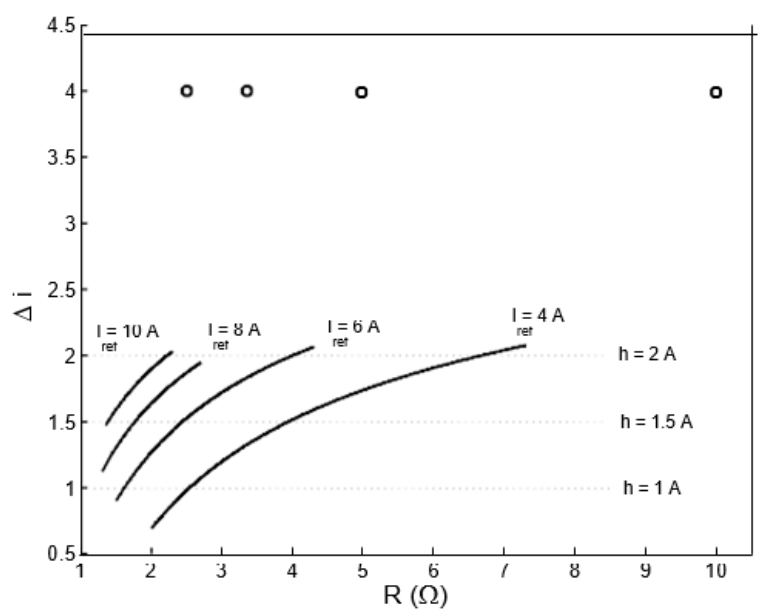

Figure 22. The corrugations $\Delta i$ of the Boost converter with the classical method (strong strokes - particular case circled) and the control with sliding mode (dotted) for $E=5 \mathrm{~V}$
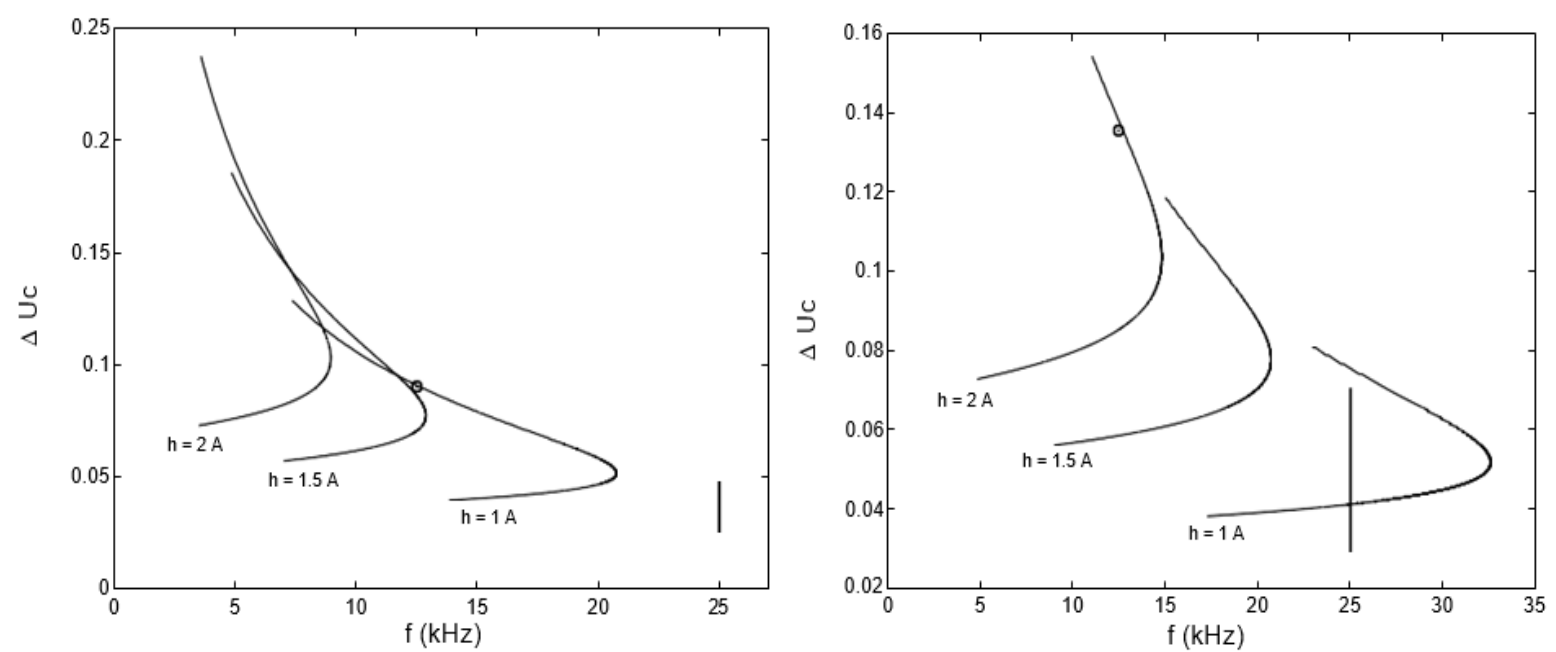

Figure 23. The switching frequency $f$ of the Boost converter with the classical method (sharp lines - particular case circled) and the sliding mode control (fine lines) for $R=2 \Omega\left(I_{\text {ref }}=4\right.$ A for the figure on the left and $I_{\text {ref }}$ $=6 \mathrm{~A}$ for the figure on the right)
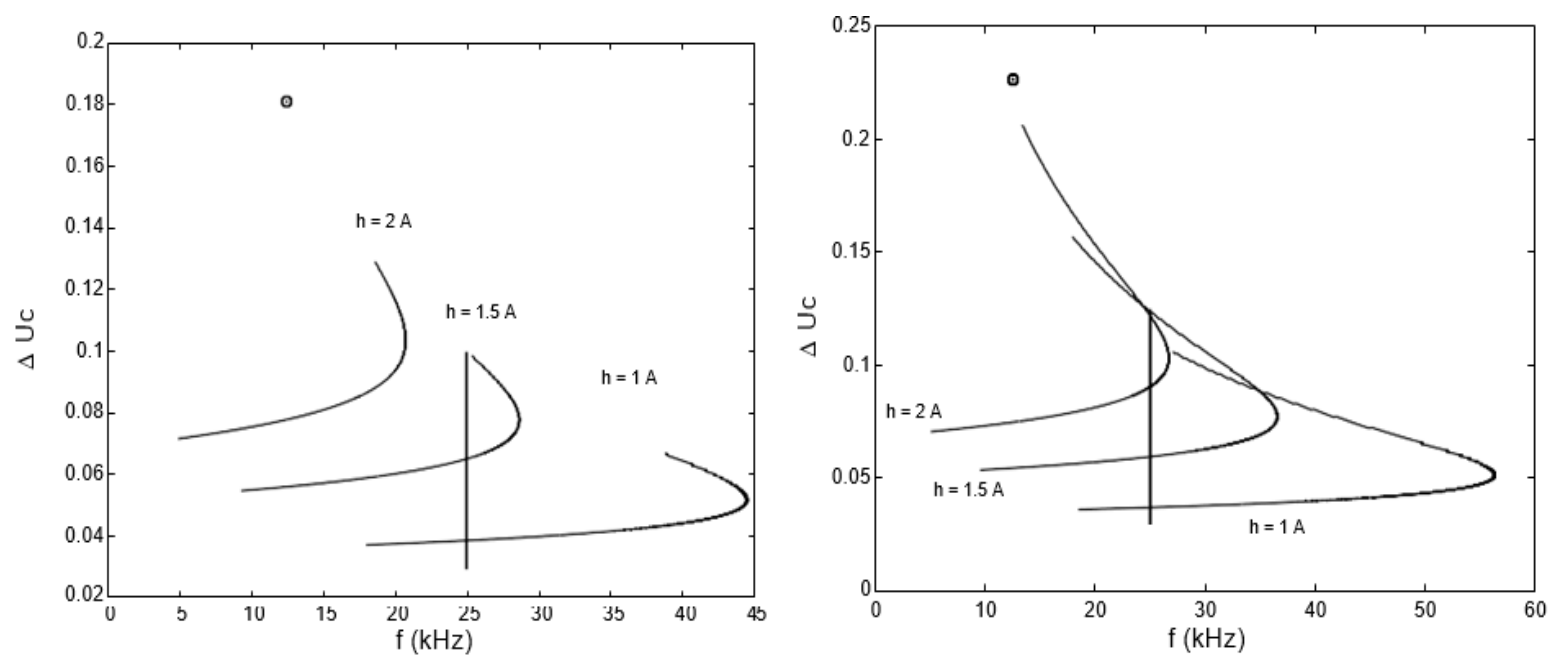

Figure 24. The switching frequency $\mathrm{f}$ of the Boost converter with the classical method (sharp lines - particular case circled) and the sliding mode control (fine lines) for $R=8 \Omega\left(I_{\text {ref }}=4 \mathrm{~A}\right.$ for the figure on the left and $I_{\text {ref }}$ $=10 \mathrm{~A}$ for the number on the right) 


\section{Conclusion}

In this paper, we have taken the example of a current-controlled Boost Converter whose chaos we eliminated (now when the duty cycle is more significant than 0.5), applying the sliding mode control.

We then developed an iterative application that provides the mathematical expressions of the output voltage $\Delta v, \Delta i$ of the inductance current, and the switching frequency, when the output is periodic (cycle ratio less than or equal to 0.5 ) for both control methods. We can thus determine which way gives the best results: it appears that the choice between the classical and the sliding mode depends strongly on the domain of variation of the parameters $E, R$, and $I_{\text {ref }}$.

\section{References}

[1]. A. Barzin, A. Sadeghieh, H. Khademi Zare, and M. Honarvar, "Hybrid Bio-Inspired Clustering Algorithm for Energy Efficient Wireless Sensor Networks," Journal of Information Technology Management, Vol.11, no.1, pp. 76-101, 2019.

[2]. Behnam Kia, F.John, Lindner and L. William Ditto, "Nonlinear dynamics based digital logic and circuits, Front. Comput. Neurosci," https://doi.org/10.3389/fncom.2015.00049, 2015.

[3]. J.L.Chabert, and A.D. Domenico, "Chaos and determinism: The News Ideas of Poincare", Paris Editions du Seuil., 1992.

[4]. W.C.Y.Chan and C.K. Tse, "Study of bifurcations in DC-DC current-program Boos converters: From quasi-periodicity to period-doubling," IEEE Transactions on Circuits and Systems -I: Fundamental Theory and Application, Vol. 44, No.12, pp.1129-1142, 1997.

[5]. Cristina Morel, "Analysis and control of chaotic dynamics, application to non-linear electronic circuits", PhD Thesis., Institute of Engineering Sciences and Techniques (ISTIA) of the University of Angers, France. (2005)

[6]. R.J. Field and M. Burger, "Oscillations and traveling waves in chemical systems", NewYork,Wiley, 1995.

[7]. M. Lellis Thivagar, and Abdulsattar Abdullah Hamad, " Topological geometry analysis forcomplex dynamic systems based on the adaptive control method," Periodicals of Engineering and Natural Sciences, Vol. 7, No.3, pp.1345-1353, 2019.

[8]. M. Lellis Thivagar, and Abdulsattar Abdullah Hamad, "Conforming Dynamics in the Metric Spaces," Journal of Information Science and Engineering, Vol 36, No 3,pp 279-291 2020.

[9]. C. Louembet, D. Arzelier, and G. Deaconu, "Robust rendezvous under maneuver execution errors", Technical Report LAAS-CNRS $N^{\circ}$ 13136, Journal of Guidance, Control and Dynamics, Vol.38, No.1, pp. 76-93, 2015.

[10]. Benjamin Durakovic, Selma Mesetovic, "Thermal Performances of Glazed Energy Storage Systems with Various Storage Materials: An Experimental study", Sustainable Cities and Society, Vol. 45, pp 422-430 (2019).

[11]. Thivagar, M. L., \& Abdullah Hamad, A. a theoretical implementation for a Proposed Hyper-Complex Chaotic System. Journal of Intelligent \& Fuzzy Systems, 2019 . (Preprint), 1-6.

[12]. R. Moore, "Study of neural networks as chaotic dynamic systems," Technical report, Macquarie University, 1999.

[13]. H. Tziperman Scher, S.E. Zebiak, and M.A. Cane, "Spatiotemporal Controlling Chaos in a realistic el nin o prediction model”, Physical Review Letters, Vol. 79, pp. 1034-1037,1997. 
[14]. V. Utkin, J. Gardner and J. Shi, "Sliding Mode Control in Electromechanical Systems," London, Taylor-Francis. 1999.

[15]. O. Woywode, H. Guldner, A.L. Baranowski and W. Schwarz, "Bifurcation and statistical analysis of DC-DC converters," IEEE Transactions on Circuits and Systems-I: Fundamental Theory and Application, Vol.50, No.8, pp. 1072-1080, 2003.

[16]. B. Durakovic, Yıldız, G., and Yahia, M. E., "Comparatıve performance evaluation of conventional and renewable thermal insulation materials used in building envelops", Tehnicki vjesnik - Technical Gazette, vol. 27, p. In Press, 2020. 